\title{
SUBGROUP FAMILIES CONTROLLING $P$-LOCAL FINITE GROUPS
}

\author{
C. BROTO, N. CASTELLANA, J. GRODAL, R. LEVI, AND B. OLIVER
}

\begin{abstract}
A $p$-local finite group consists of a finite $p$-group $S$, together with a pair of categories which encode "conjugacy" relations among subgroups of $S$, and which are modelled on the fusion in a Sylow $p$-subgroup of a finite group. It contains enough information to define a classifying space which has many of the same properties as $p$-completed classifying spaces of finite groups. In this paper, we examine which subgroups control this structure. More precisely, we prove that the question of whether an abstract fusion system $\mathcal{F}$ over a finite $p$-group $S$ is saturated can be determined by just looking at smaller classes of subgroups of $S$. We also prove that the homotopy type of the classifying space of a given $p$-local finite group is independent of the family of subgroups used to define it, in the sense that it remains unchanged when that family ranges from the set of $\mathcal{F}$-centric $\mathcal{F}$-radical subgroups (at a minimum) to the set of $\mathcal{F}$-quasicentric subgroups (at a maximum). Finally, we look at constrained fusion systems, analogous to $p$-constrained finite groups, and prove that they in fact all arise from groups.
\end{abstract}

A $p$-local finite group consists of a finite $p$-group $\mathrm{S}$, together with a pair of categories $(\mathcal{F}, \mathcal{L})$, of which $\mathcal{F}$ is modeled on the conjugacy (or fusion) in a Sylow subgroup of a finite group. The category $\mathcal{L}$ is essentially an extension of $\mathcal{F}$ and contains just enough extra information so that its $p$-completed nerve has many of the same properties as $p$-completed classifying spaces of finite groups. The main purpose of this paper is to study when the set of objects of $\mathcal{F}$ or $\mathcal{L}$ can be changed without changing the conjugacy encoded by $\mathcal{F}$ or the homotopy type of the nerve of $\mathcal{L}$. The tools introduced simplify the construction and manipulation of $p$-local finite groups in many cases.

We first recall the fusion and linking categories associated to a finite group. Fix a prime $p$, a finite group $G$, and a Sylow $p$-subgroup $S$ of $G$. A $p$-fusion category for $G$ is a category $\mathcal{F}=\mathcal{F}_{S}^{\mathcal{H}}(G)$, whose object set is a set $\mathcal{H}$ of subgroups of $S$, and whose morphisms are the homomorphisms between subgroups in $\mathcal{H}$ induced by conjugation in $G$. The associated linking category $\mathcal{L}=\mathcal{L}_{S}^{\mathcal{H}}(G)$ has the same objects, and morphisms from $P$ to $Q$ are given by the formula

$$
\operatorname{Mor}_{\mathcal{L}}(P, Q)=\left\{x \in G \mid x P x^{-1} \leq Q\right\} / O^{p}\left(C_{G}(P)\right) .
$$

Here, $O^{p}(-)$ is the subgroup generated by elements of order prime to $p$. There is a canonical quotient functor $\mathcal{L}_{S}^{\mathcal{H}}(G) \longrightarrow \mathcal{F}_{S}^{\mathcal{H}}(G)$ which sends the class of $x$ to

2000 Mathematics Subject Classification Primary 55R35. Secondary 55R40, 20D20.

C. Broto and N. Castellana were partially supported by MCYT grant BFM2001-2035

J. Grodal was partially supported by NSF grant DMS-0104318

R. Levi was partially supported by EPSRC grant GR/M7831

B. Oliver was partially supported by UMR 7539 of the CNRS 
conjugation by $x$. It was shown in $\left[4\right.$ that the homotopy theory of the nerve $\left|\mathcal{L}_{S}^{\mathcal{H}}(G)\right|$ (for the right choice of $\mathcal{H}$ ) is closely related to the $p$-local homotopy theory of $B G$.

Fusion and linking categories were designed to a large extent to capture the " $p$ local structure" of finite groups, blocks, and $p$-completed classifying spaces in a way which does not depend directly on the structure of the ambient group. Many results in group theory, such as Alperin's fusion theorem [1] and the work by Alperin and Broué on fusion in block theory [2], can be formulated in terms of fusion categories. One is thus led to search for an axiomatic definition of these concepts. The definition of a saturated fusion system $\mathcal{F}$ over a $p$-group $S$, generalizing $p$-fusion categories of finite groups, was first given by Puig [18. A simplified (but equivalent) definition of a saturated fusion system, along with an axiomatic definition of a "centric" linking system, was later given in [5, §1]. Here, the word "centric" refers to the set of objects in the linking system, which will be described in Section 1 A $p$-local finite group is then defined to be a triple $(S, \mathcal{F}, \mathcal{L})$, where $S$ is a finite $p$-group, $\mathcal{F}$ is a saturated fusion system over $S$, and $\mathcal{L}$ is a centric linking system associated to $\mathcal{F}$. The classifying space of such a triple is the $p$-completed nerve $|\mathcal{L}|_{p}^{\wedge}$. For any $S \leq G$ as above, $\left(S, \mathcal{F}_{S}(G), \mathcal{L}_{S}^{c}(G)\right.$ ) (where the categories are taken for appropriate families of subgroups) is a $p$-local finite group with classifying space $\left|\mathcal{L}_{S}^{c}(G)\right|_{p}^{\wedge} \simeq B G_{p}^{\wedge}$.

The main goal of this paper is to examine the role of the set $\mathcal{H}$ of subgroups of $S$ on which the fusion and linking systems are defined; i.e., to show when the set can be changed without changing $\mathcal{F}$ and $\mathcal{L}$ in an "essential" way. Related questions have been studied extensively when $\mathcal{F}$ comes from a finite group $G$, both in connection with the Alperin's fusion theorem (cf. 1] and 17]) and more indirectly in connection with the study of homology decompositions (cf. 8 and 11]). In a subsequent paper [6], we use the tools developed in this paper to study the extension theory of fusion systems and $p$-local finite groups, in part motivated by our desire to develop more ways of constructing $p$-local finite groups that do not come from groups. Such "exotic" $p$-local finite groups do exist for all primes, and examples are given in $\mathbf{5}$ $\S 9]$, 21, 15, and [7, but we still have no really good tools for constructing them, nor any sense of how frequently they occur.

We now describe the results of the paper in more detail. We refer the reader to Section 11 for the definitions of abstract saturated fusion systems and centric linking systems; and also of $\mathcal{F}$-centric and $\mathcal{F}$-radical subgroups for a fusion system $\mathcal{F}$ (analogous to the usual concepts of $p$-centric subgroups and radical $p$-subgroups of a finite group). However, the precise definitions will not be essential to follow this introductory discussion. We also refer the reader to the end of the introduction for a list of notation which will be used throughout the paper.

One of the most difficult problems, when constructing exotic fusion systems, is showing that the fusion system one has constructed satisfies the axioms of saturation (see Definition 1.3). This job is clearly simpler if one only needs to check the axioms on subgroups which are centric, rather than having to do so on all subgroups. The following theorem is used several times in our paper [6], and can be used to shorten the proof of saturation of the exotic fusion systems in [5 59 .

TheOREM A. Let $\mathcal{F}$ be a fusion system over a finite p-group $S$, and assume that all morphisms in $\mathcal{F}$ are composites of restrictions of morphisms between $\mathcal{F}$-centric 
subgroups. If $\mathcal{F}$ satisfies the axioms of saturation (Definition 1.3) when applied to $\mathcal{F}$-centric subgroups of $S$, then $\mathcal{F}$ is saturated.

This theorem is stated more precisely, and in greater generality, in Theorem 2.2 There, we replace "F-F-centric" subgroups in the above formulation by "any collection of subgroups containing all subgroups which are both $\mathcal{F}$-centric and $\mathcal{F}$-radical and is closed under $\mathcal{F}$-conjugacy"; but at the price of an additional hypothesis. As such, it can be thought of as a converse to Alperin's fusion theorem for abstract fusion systems (as shown in [18,2.13] and [5 Theorem A.10]), which says that if $\mathcal{F}$ is a saturated fusion system, then it is generated by restrictions of automorphisms of $\mathcal{F}$-centric $\mathcal{F}$-radical subgroups.

In many applications, it is useful to construct linking systems with respect to different sets of subgroups than the $\mathcal{F}$-centric subgroups of $S$. If $G$ is a finite group, then we call a $p$-subgroup $P \leq G$-quasicentric if $O^{p}\left(C_{G}(P)\right)$ has order prime to $p$; equivalently, if $B C_{G}(P)_{p}^{\wedge}$ is the classifying space of some $p$-group. When $\mathcal{F}$ is a saturated fusion system over a $p$-group $S$, then we make an analogous definition of an $\mathcal{F}$-quasicentric subgroup of $S$ in Section 3 . When $\mathcal{F}$ is the fusion system of a block $b$ with defect group $S$, then the $\mathcal{F}$-quasicentric subgroups of $S$ correspond to the nil-centralized pointed groups, in the sense of Puig [19, which are associated to $b$.

Our next theorem shows that the homotopy type of the classifying space of a $p$-local finite group $(S, \mathcal{F}, \mathcal{L})$ is also determined by a linking system based on any set of $\mathcal{F}$-quasicentric subgroups of $S$ which contains at least those which are both $\mathcal{F}$-centric and $\mathcal{F}$-radical. This result can also be interpreted as a statement about homology decompositions for $p$-local finite groups, and as such is motivated by [8, 1.20] and [11, Theorem 1.5]. It is restated and proved as Theorem 3.5] and is essential when studying "extensions" of $p$-local finite groups with $p$-group quotient in [6].

TheOREm B. Let $(S, \mathcal{F}, \mathcal{L})$ be a p-local finite group. Then there exists a category $\mathcal{L}^{q}$ containing $\mathcal{L}$ as a full subcategory, whose objects are the $\mathcal{F}$-quasicentric subgroups of $S$, and such that the inclusion of nerves $|\mathcal{L}| \subseteq\left|\mathcal{L}^{q}\right|$ is a homotopy equivalence. Furthermore, if $\mathcal{H}$ is any collection of $\mathcal{F}$-quasicentric subgroups of $S$ containing all $P \leq S$ which are both $\mathcal{F}$-centric and $\mathcal{F}$-radical, and $\mathcal{L}^{\mathcal{H}} \subseteq \mathcal{L}^{q}$ is the full subcategory whose objects are the subgroups in $\mathcal{H}$, then the inclusions of $\mathcal{L}^{\mathcal{H}}$ and $\mathcal{L}$ in $\mathcal{L}^{q}$ induce homotopy equivalences

$$
\left|\mathcal{L}^{\mathcal{H}}\right| \simeq\left|\mathcal{L}^{q}\right| \simeq|\mathcal{L}|
$$

We conclude this paper, in Section 4 with a very specialized family of examples: fusion systems whose entire structure is controlled by a single $p$-subgroup. If $G$ is a finite group which has no nontrivial normal subgroup of order prime to $p$, then $G$ is called $p$-constrained if there is a normal $p$-subgroup $P \triangleleft G$ such that $C_{G}(P) \leq P$; equivalently, such that $G / P$ can be identified (via conjugation) with a subgroup of Out $(P)$. In Section 4 we give an analogous definition of a constrained fusion system (Definition 4.1), and then prove the following proposition (restated as Proposition 4.3). 
Proposition C. Let $\mathcal{F}$ be a constrained saturated fusion system over a finite p-group $S$. Then there exists a unique $p^{\prime}$-reduced $p$-constrained finite group $G$ such that $\mathcal{F}=\mathcal{F}_{S}(G)$.

For easy reference, we end the introduction with a list of notation and terminology which is used throughout the paper.

- $\operatorname{Syl}_{p}(G)$ denotes the set of Sylow $p$-subgroups of $G$.

- $O_{p}(G)$ is the maximal normal $p$-subgroup of $G$.

- $O_{p^{\prime}}(G)$ is the maximal normal subgroup of $G$ of order prime to $p$.

- $G$ is $p$-reduced ( $p^{\prime}$-reduced) if $O_{p}(G)=1$ (if $O_{p^{\prime}}(G)=1$ ).

- $O^{p}(G)$ the minimal normal subgroup of $G$ of $p$-power index.

- $N_{G}(P, Q)=\left\{x \in G \mid x P x^{-1} \leq Q\right\} \quad$ (for $\left.P, Q \leq G\right)$.

- $c_{x}$ denotes conjugation by $x\left(g \mapsto x g x^{-1}\right)$.

- $N_{S}^{K}(P)=\left\{x \in N_{S}(P) \mid c_{x} \in K\right\} \quad($ for $P \leq S$ and $K \leq \operatorname{Aut}(P))$.

- $\operatorname{Hom}_{G}(H, K)$ (for $H, K \leq G$ ) is the set of homomorphisms from $H$ to $K$ induced by conjugation in $G$.

- $\operatorname{Aut}_{G}(H)=\operatorname{Hom}_{G}(H, H)$, and $\operatorname{Out}_{G}(H)=\operatorname{Aut}_{G}(H) / \operatorname{Inn}(H)$.

- In a fusion system $\mathcal{F}, \operatorname{Hom}_{\mathcal{F}}(P, Q)=\operatorname{Mor}_{\mathcal{F}}(P, Q), \operatorname{Iso}_{\mathcal{F}}(P, Q)=\operatorname{Hom}_{\mathcal{F}}(P, Q)$ if $|P|=|Q|, \operatorname{Aut}_{\mathcal{F}}(P)=\operatorname{Iso}_{\mathcal{F}}(P, P)$, and $\operatorname{Out}_{\mathcal{F}}(P)=\operatorname{Aut}_{\mathcal{F}}(P) / \operatorname{Inn}(P)$.

All five authors would like to thank the University of Aberdeen, the University of Paris 13 and the CRM in Barcelona for giving us the opportunity on several occasions to get together while doing this work.

\section{A quick review of p-local finite groups}

We first recall the definitions of a fusion system, and a saturated fusion system, in the form given in [5].

Definition 1.1 [18 and [5 Definition 1.1]. A fusion system over a finite pgroup $S$ is a category $\mathcal{F}$, where $\operatorname{Ob}(\mathcal{F})$ is the set of all subgroups of $S$, and which satisfies the following two properties for all $P, Q \leq S$ :

- $\operatorname{Hom}_{S}(P, Q) \subseteq \operatorname{Hom}_{\mathcal{F}}(P, Q) \subseteq \operatorname{Inj}(P, Q)$; and

- each $\varphi \in \operatorname{Hom}_{\mathcal{F}}(P, Q)$ is the composite of an isomorphism in $\mathcal{F}$ followed by an inclusion.

We next specify certain collections of subgroups relative to a given fusion system. If $\mathcal{F}$ is a fusion system over a finite $p$-subgroup $S$, then two subgroups $P, Q \leq S$ are said to be $\mathcal{F}$-conjugate if they are isomorphic as objects of the category $\mathcal{F}$. 
DeFinition 1.2. Let $\mathcal{F}$ be a fusion system over a finite p-subgroup $S$.

- A subgroup $P \leq S$ is $\mathcal{F}$-centric if $C_{S}\left(P^{\prime}\right)=Z\left(P^{\prime}\right)$ for all $P^{\prime} \leq S$ which are $\mathcal{F}$-conjugate to $P$.

- A subgroup $P \leq S$ is $\mathcal{F}$-radical if $\operatorname{Out}_{\mathcal{F}}(P)$ is p-reduced; i.e., if $O_{p}\left(\operatorname{Out}_{\mathcal{F}}(P)\right)=1$.

If $\mathcal{F}=\mathcal{F}_{S}(G)$ for some finite group $G$, then $P \leq S$ is $\mathcal{F}$-centric if and only if $P$ is $p$-centric in $G$ (i.e., $Z(P) \in \operatorname{Syl}_{p}\left(C_{G}(P)\right)$ ), and $P$ is $\mathcal{F}$-radical if and only if $N_{G}(P) / P \cdot C_{G}(P)$ is $p$-reduced.

The following additional definitions and conditions are needed in order for these systems to be very useful.

Definition 1.3 [8], see 5 Definition 1.2]. Let $\mathcal{F}$ be a fusion system over a p-group $S$.

- A subgroup $P \leq S$ is fully centralized in $\mathcal{F}$ if $\left|C_{S}(P)\right| \geq\left|C_{S}\left(P^{\prime}\right)\right|$ for all $P^{\prime} \leq S$ which is $\mathcal{F}$-conjugate to $P$.

- A subgroup $P \leq S$ is fully normalized in $\mathcal{F}$ if $\left|N_{S}(P)\right| \geq\left|N_{S}\left(P^{\prime}\right)\right|$ for all $P^{\prime} \leq S$ which is $\mathcal{F}$-conjugate to $P$.

- $\mathcal{F}$ is a saturated fusion system if the following two conditions hold:

(I) For all $P \leq S$ which is fully normalized in $\mathcal{F}, P$ is fully centralized in $\mathcal{F}$ and $\operatorname{Aut}_{S}(P) \in \operatorname{Syl}_{p}\left(\operatorname{Aut}_{\mathcal{F}}(P)\right)$.

(II) If $P \leq S$ and $\varphi \in \operatorname{Hom}_{\mathcal{F}}(P, S)$ are such that $\varphi P$ is fully centralized, and if we set

$$
N_{\varphi}=\left\{g \in N_{S}(P) \mid \varphi c_{g} \varphi^{-1} \in \operatorname{Aut}_{S}(\varphi P)\right\},
$$

then there is $\bar{\varphi} \in \operatorname{Hom}_{\mathcal{F}}\left(N_{\varphi}, S\right)$ such that $\left.\bar{\varphi}\right|_{P}=\varphi$.

If $G$ is a finite group and $S \in \operatorname{Syl}_{p}(G)$, then the category $\mathcal{F}_{S}(G)$ defined in the introduction is a saturated fusion system (see [5. Proposition 1.3]).

We now turn to linking systems associated to abstract fusion systems.

Definition 1.4 [5] Definition 1.7]. Let $\mathcal{F}$ be a fusion system over the p-group $S$. A centric linking system associated to $\mathcal{F}$ is a category $\mathcal{L}$ whose objects are the $\mathcal{F}$-centric subgroups of $S$, together with a functor $\pi: \mathcal{L} \longrightarrow \mathcal{F}^{c}$, and "distinguished" monomorphisms $P \stackrel{\delta_{P}}{\longrightarrow} \operatorname{Aut}_{\mathcal{L}}(P)$ for each $\mathcal{F}$-centric subgroup $P \leq S$, which satisfy the following conditions.

(A) $\pi$ is the identity on objects. For each pair of objects $P, Q \in \mathcal{L}, Z(P)$ acts freely on $\operatorname{Mor}_{\mathcal{L}}(P, Q)$ by composition (upon identifying $Z(P)$ with $\delta_{P}(Z(P)) \leq$ $\left.\operatorname{Aut}_{\mathcal{L}}(P)\right)$, and $\pi$ induces a bijection

$$
\operatorname{Mor}_{\mathcal{L}}(P, Q) / Z(P) \stackrel{\cong}{\longrightarrow} \operatorname{Hom}_{\mathcal{F}}(P, Q) \text {. }
$$

(B) For each $\mathcal{F}$-centric subgroup $P \leq S$ and each $x \in P, \pi\left(\delta_{P}(x)\right)=c_{x} \in$ $\operatorname{Aut}_{\mathcal{F}}(P)$. 
(C) For each $f \in \operatorname{Mor}_{\mathcal{L}}(P, Q)$ and each $x \in P, f \circ \delta_{P}(x)=\delta_{Q}(\pi f(x)) \circ f$.

A $p$-local finite group is defined to be a triple $(S, \mathcal{F}, \mathcal{L})$, where $S$ is a finite $p$-group, $\mathcal{F}$ is a saturated fusion system over $S$, and $\mathcal{L}$ is a centric linking system associated to $\mathcal{F}$. The classifying space of the triple $(S, \mathcal{F}, \mathcal{L})$ is the $p$-completed nerve $|\mathcal{L}|_{p}^{\wedge}$.

For any finite group $G$ with Sylow $p$-subgroup $S$, the category $\mathcal{L}_{S}^{c}(G)$ defined in the introduction is easily seen to be a centric linking system associated to $\mathcal{F}_{S}(G)$. Thus $\left(S, \mathcal{F}_{S}(G), \mathcal{L}_{S}^{c}(G)\right)$ is a $p$-local finite group, with classifying space $\left|\mathcal{L}_{S}^{c}(G)\right|_{p}^{\wedge} \simeq$ $B G_{p}^{\wedge}$ (see [4, Proposition 1.1]).

The following definitions are somewhat more specialized, and are translations to the setting of fusion systems of the concepts of a normal $p$-subgroup of a finite group, and of strongly and weakly closed subgroups.

DeFInITIOn 1.5. Let $\mathcal{F}$ be a saturated fusion system over a p-group $S$. Then for any normal subgroup $Q \triangleleft S$,

(a) $Q$ is strongly closed in $\mathcal{F}$ if no element of $Q$ is $\mathcal{F}$-conjugate to an element of $S \backslash Q$;

(b) $Q$ is weakly closed in $\mathcal{F}$ if no other subgroup of $S$ is $\mathcal{F}$-conjugate to $Q$; and

(c) $Q$ is normal in $\mathcal{F}$ if each morphism $\alpha \in \operatorname{Hom}_{\mathcal{F}}\left(P, P^{\prime}\right)$ in $\mathcal{F}$ extends to some $\bar{\alpha} \in \operatorname{Hom}_{\mathcal{F}}\left(P Q, P^{\prime} Q\right)$ such that $\bar{\alpha}(Q)=Q$.

Equivalently, $Q \triangleleft S$ is normal in $\mathcal{F}$ if and only if the normalizer fusion system $N_{\mathcal{F}}(Q)$ is equal to $\mathcal{F}$ as fusion systems over $S$ (see [5, Definition 6.1]). The next proposition, which is motivated by [17. Proposition IV.2], gives two equivalent conditions for a subgroup to be normal in $\mathcal{F}$.

Proposition 1.6. Let $\mathcal{F}$ be a fusion system over $S$. Then the following conditions on a subgroup $Q \leq S$ are equivalent:

(a) $Q$ is normal in $\mathcal{F}$.

(b) $Q$ is strongly closed in $\mathcal{F}$ and is contained in all $\mathcal{F}$-radical subgroups of $S$.

(c) $Q$ is weakly closed in $\mathcal{F}$ and is contained in all $\mathcal{F}$-radical subgroups of $S$.

Proof. Assume first that $Q$ is normal in $\mathcal{F}$. In particular, if an element $x \in Q$ is $\mathcal{F}$-conjugate to an element $y \in S \backslash Q$, then the isomorphism in $\mathcal{F}$ from $\langle x\rangle$ to $\langle y\rangle$ extends to a morphism $Q=\langle Q, x\rangle \longrightarrow\langle Q, y\rangle$. But such a morphism clearly cannot send $Q$ to itself. Thus $Q$ is strongly closed in $\mathcal{F}$. If $P \leq S$ does not contain $Q$, then $N_{P Q}(P) / P$ is a nontrivial $p$-subgroup of $\operatorname{Out}_{\mathcal{F}}(P)$, which is in fact normal there. To see normality notice that if $\alpha \in \operatorname{Aut}_{\mathcal{F}}(P)$ then $\alpha$ extends to $\bar{\alpha} \in \operatorname{Aut}_{\mathcal{F}}(P Q)$ since $Q$ normal in $\mathcal{F}$, so for all $x \in N_{P Q}(P)$ we have $\alpha c_{x} \alpha^{-1}=\left(\bar{\alpha} c_{x} \bar{\alpha}^{-1}\right)_{\mid P}=c_{\bar{\alpha}(x)} \in$ $\operatorname{Aut}_{P Q}(P)$. Hence such a subgroup $P$ cannot be $\mathcal{F}$-radical. Thus, all $\mathcal{F}$-radical subgroups of $S$ contain $Q$. This shows (a) $\Rightarrow$ (b).

Condition (b) clearly implies (c), and so it remains to show (c) $\Rightarrow$ (a). Assume that $Q$ is weakly closed in $\mathcal{F}$, and that all $\mathcal{F}$-radical subgroups contain $Q$. Then by 
Alperin's fusion theorem, each morphism in $\mathcal{F}$ is a composite of morphisms, each of which is the restriction of a morphism between subgroups containing $Q$, and which necessarily sends $Q$ to itself (since $Q$ is weakly closed). In other words, each $\varphi \in \operatorname{Hom}_{\mathcal{F}}\left(P, P^{\prime}\right)$ extends to a morphism $\bar{\varphi} \in \operatorname{Hom}_{\mathcal{F}}\left(P Q, P^{\prime} Q\right)$ which sends $Q$ to itself, and hence $Q$ is normal in $\mathcal{F}$.

\section{Centric and radical subgroups determine saturation}

Given a fusion system which is not known to come from a group (or a block), it turns out to be difficult in general to show that it is saturated when using the definition directly. This is one of the obstacles one encounters when trying to construct $p$-local finite groups which do not come from groups.

The main result of this section, Theorem 2.2 says that it suffices to check the axioms of saturation on the centric subgroups, in the sense that any fusion system which satisfies these axioms for its centric subgroups generates a saturated fusion system in a way made precise below. In fact, our result is stronger than that. We prove that it suffices to check the axioms of saturation on those subgroups which are centric and radical, and a much weaker condition on the centric subgroups which are not radical.

Before stating the main results, we make some definitions.

Definition 2.1. Let $\mathcal{F}$ be any fusion system over a finite p-group $S$, and let $\mathcal{H}$ be a set of subgroups of $S$ closed under $\mathcal{F}$-conjugacy.

(a) $\mathcal{F}$ is $\mathcal{H}$-generated if every morphism in $\mathcal{F}$ is a composite of restrictions of morphisms in $\mathcal{F}$ between subgroups in $\mathcal{H}$.

(b) $\mathcal{F}$ is $\mathcal{H}$-saturated if conditions (I) and (II) hold in $\mathcal{F}$ for all subgroups $P \in \mathcal{H}$.

In terms of these definitions, Alperin's fusion theorem for abstract fusion systems (in the form shown in [5. Theorem A.10]) can be reformulated by saying that if $\mathcal{F}$ is a saturated fusion system over $S$, and $\mathcal{H}$ is the family of $\mathcal{F}$-centric, $\mathcal{F}$-radical subgroups of $S$, then $\mathcal{F}$ is $\mathcal{H}$-generated.

Our main result in this section can be thought of as a converse to this form of the fusion theorem. In practice, it often simplifies the task of deciding whether a fusion system is saturated or not. As one example, the proof of [5. Proposition 9.1] - the proof that the fusion systems constructed there are saturated - becomes far simpler when we can use Theorem [2.2] applied with $\mathcal{H}$ the set of $\mathcal{F}$-centric subgroups of $S$.

TheOREm 2.2. Let $\mathcal{F}$ be a fusion system over a finite p-group $S$, and let $\mathcal{H}$ be a set of subgroups of $S$ closed under $\mathcal{F}$-conjugacy that contains all $\mathcal{F}$-centric, $\mathcal{F}$-radical subgroups of $S$. Assume that $\mathcal{F}$ is $\mathcal{H}$-generated and $\mathcal{H}$-saturated, and that

$(*)$ each $\mathcal{F}$-conjugacy class of subgroups of $S$ which are $\mathcal{F}$-centric but not in $\mathcal{H}$ contains at least one subgroup $P$ such that $\operatorname{Out}_{S}(P) \cap O_{p}\left(\operatorname{Out}_{\mathcal{F}}(P)\right) \neq 1$.

Then $\mathcal{F}$ is saturated. 
Note that the condition that $\mathcal{H}$ contain all $\mathcal{F}$-centric, $\mathcal{F}$-radical subgroups of $S$ is implied by $(*)$; but we keep it in the statement for the sake of emphasis. Condition $(*)$ might at first sight seem artificial, but we construct an example at the end of this section showing that it is, in fact, necessary, and not implied by the other hypotheses of the theorem.

We first discuss the relation between conditions (I) and (II) in Definition 1.3 and certain other, similar conditions on fusion systems. We recall the definition of $N_{\varphi}$ for any given $\varphi \in \operatorname{Mor}_{\mathcal{F}}(P, Q)$,

$$
N_{\varphi}=\left\{x \in N_{S}(P) \mid \varphi c_{x} \varphi^{-1} \in \operatorname{Aut}_{S}(\varphi(P))\right\} .
$$

LEMma 2.3. Let $\mathcal{F}$ be a fusion system over a p-group $S$, and let $\mathcal{H}$ be a set of subgroups of $S$ closed under $\mathcal{F}$-conjugacy. Consider the following conditions on $\mathcal{F}$ :

$(\mathrm{I})_{\mathcal{H}}:$ For each subgroup $P \in \mathcal{H}$ fully normalized in $\mathcal{F}, P$ is fully centralized and $\operatorname{Aut}_{S}(P) \in \operatorname{Syl}_{p}\left(\operatorname{Aut}_{\mathcal{F}}(P)\right)$.

$\left(\mathrm{I}^{\prime}\right)_{\mathcal{H}}:$ Each $P \in \mathcal{H}$ is $\mathcal{F}$-conjugate to a fully centralized subgroup $P^{\prime} \in \mathcal{H}$ such that $\operatorname{Aut}_{S}\left(P^{\prime}\right) \in \operatorname{Syl}_{p}\left(\operatorname{Aut}_{\mathcal{F}}\left(P^{\prime}\right)\right)$.

$(\mathrm{II})_{\mathcal{H}}:$ For each $P \in \mathcal{H}$, and each $\varphi \in \operatorname{Hom}_{\mathcal{F}}(P, S)$ such that $\varphi(P)$ is fully centralized in $\mathcal{F}, \varphi$ extends to a morphism $\bar{\varphi} \in \operatorname{Hom}_{\mathcal{F}}\left(N_{\varphi}, S\right)$.

$(\mathrm{IIA})_{\mathcal{H}}:$ Each $\mathcal{F}$-conjugacy class $\mathcal{P} \subseteq \mathcal{H}$ contains a fully normalized subgroup $\widehat{P} \in$ $\mathcal{P}$ with the following property: for all $P \in \mathcal{P}$, there exists a morphism $\varphi \in$ $\operatorname{Hom}_{\mathcal{F}}\left(N_{S}(P), N_{S}(\widehat{P})\right)$ such that $\varphi(P)=\widehat{P}$.

(IIB) $)_{\mathcal{H}}$ : For each fully normalized subgroup $\widehat{P} \in \mathcal{H}$ and each $\varphi \in \operatorname{Aut}_{\mathcal{F}}(\widehat{P})$, there is a morphism $\bar{\varphi} \in \operatorname{Hom}_{\mathcal{F}}\left(N_{\varphi}, N_{S}(\widehat{P})\right)$ which extends $\varphi$.

Then

(a) $(\mathrm{I})_{\mathcal{H}} \Longleftrightarrow\left(\mathrm{I}^{\prime}\right)_{\mathcal{H}} ;$ and

(b) $(\mathrm{I})_{\mathcal{H}}+(\mathrm{II})_{\mathcal{H}} \Longrightarrow(\mathrm{IIA})_{\mathcal{H}}+(\mathrm{IIB})_{\mathcal{H}} \Longrightarrow(\mathrm{II})_{\mathcal{H}}$.

Proof. (a) Condition $(\mathrm{I})_{\mathcal{H}}$ clearly implies $\left(\mathrm{I}^{\prime}\right)_{\mathcal{H}}$, since every $P \leq S$ is $\mathcal{F}$-conjugate to a fully normalized subgroup. To see the converse, assume $P \in \mathcal{H}$ is fully normalized. By $\left(\mathrm{I}^{\prime}\right)_{\mathcal{H}}$ we can choose $P^{\prime} \in \mathcal{H}$ which is $\mathcal{F}$-conjugate to $P$, fully centralized, and satisfies $\operatorname{Aut}_{S}\left(P^{\prime}\right) \in \operatorname{Syl}_{p}\left(\operatorname{Aut}_{\mathcal{F}}\left(P^{\prime}\right)\right)$. Then

$$
\begin{aligned}
\left|\operatorname{Aut}_{S}\left(P^{\prime}\right)\right| \cdot\left|C_{S}\left(P^{\prime}\right)\right| & =\left|N_{S}\left(P^{\prime}\right)\right| \leq\left|N_{S}(P)\right| \\
& =\left|\operatorname{Aut}_{S}(P)\right| \cdot\left|C_{S}(P)\right| \leq\left|\operatorname{Aut}_{S}\left(P^{\prime}\right)\right| \cdot\left|C_{S}\left(P^{\prime}\right)\right|:
\end{aligned}
$$

the first inequality holds since $P$ is fully normalized, and the second by the assumptions on $P^{\prime}$. Thus all of these inequalities are equalities, and so $P$ is fully centralized and $\operatorname{Aut}_{S}(P) \in \operatorname{Syl}_{p}\left(\operatorname{Aut}_{\mathcal{F}}(P)\right)$.

(b) Assume $(\mathrm{I})_{\mathcal{H}}$ and $(\mathrm{II})_{\mathcal{H}}$ hold; we next prove that this implies $(\mathrm{IIA})_{\mathcal{H}}$ and $(\mathrm{IIB})_{\mathcal{H}}$. We first check condition (IIB) $\mathcal{H}$. Let $\widehat{\varphi}=\iota_{\widehat{P}} \circ \varphi$ where $\iota_{\widehat{P}}$ is the inclusion of $\widehat{P}$ in $S$. Since $\widehat{P}$ is fully normalized, condition $(\mathrm{I})_{\mathcal{H}}$ implies that $\widehat{P}$ is also fully centralized. 
By condition $(\mathrm{II})_{\mathcal{H}}, \widehat{\varphi}$ extends to $\bar{\varphi} \in \operatorname{Hom}_{\mathcal{F}}\left(N_{\widehat{\varphi}}, S\right)$, where

$$
\begin{aligned}
N_{\widehat{\varphi}} & =\left\{g \in N_{S}(\widehat{P}) \mid \widehat{\varphi} c_{g} \widehat{\varphi}^{-1} \in \operatorname{Aut}_{S}(\widehat{\varphi}(\widehat{P}))\right\} \\
& =\left\{g \in N_{S}(\widehat{P}) \mid \varphi c_{g} \varphi^{-1} \in \operatorname{Aut}_{S}(\widehat{P})\right\}=N_{\varphi} .
\end{aligned}
$$

Furthermore, $\operatorname{Im}(\bar{\varphi}) \leq N_{S}(\widehat{P})$, since $\widehat{P} \triangleleft N_{\widehat{\varphi}}$.

Next we check that $(\text { IIA })_{\mathcal{H}}$ holds. Fix an $\mathcal{F}$-conjugacy class $\mathcal{P} \subseteq \mathcal{H}$, and choose a fully normalized subgroup $\widehat{P} \in \mathcal{P}$. Since $(\mathrm{I})_{\mathcal{H}}$ holds, $\widehat{P}$ is also fully centralized, and $\operatorname{Aut}_{S}(\widehat{P}) \in \operatorname{Syl}_{p}\left(\operatorname{Aut}_{\mathcal{F}}(\widehat{P})\right)$. Thus for any $P \in \mathcal{P}$ and any $\varphi \in \operatorname{Iso}_{\mathcal{F}}(P, \widehat{P})$, there exists $\chi \in \operatorname{Aut}_{\mathcal{F}}(P)$ such that $\varphi \chi \operatorname{Aut}_{S}(P) \chi^{-1} \varphi^{-1} \leq \operatorname{Aut}_{S}(\widehat{P})$. Then

$$
N_{\varphi \chi} \stackrel{\text { def }}{=}\left\{g \in N_{S}(P) \mid \varphi \chi c_{g} \chi^{-1} \varphi^{-1} \in \operatorname{Aut}_{S}(\widehat{P})\right\}=N_{S}(P),
$$

and hence the morphism $\varphi \chi$ extends to $\bar{\varphi} \in \operatorname{Hom}_{\mathcal{F}}\left(N_{S}(P), S\right)$ by (II) $)_{\mathcal{H}}$. Then $\bar{\varphi}(P)=\varphi \chi(P)=\widehat{P}$, and hence $\operatorname{Im}(\bar{\varphi}) \leq N_{S}(\widehat{P})$.

It remains to prove the last implication. Assume $(\text { IIA })_{\mathcal{H}}$ and $(\text { IIB })_{\mathcal{H}}$; we must prove $(\mathrm{II})_{\mathcal{H}}$. Fix $P \in \mathcal{H}$ and $\varphi \in \operatorname{Hom}_{\mathcal{F}}(P, S)$ such that $P^{\prime} \stackrel{\text { def }}{=} \varphi(P)$ is fully centralized in $\mathcal{F}$. Using (IIA) $)_{\mathcal{H}}$, choose a fully normalized subgroup $\widehat{P}$ which is $\mathcal{F}$-conjugate to $P, P^{\prime}$, and morphisms

$$
\psi \in \operatorname{Hom}_{\mathcal{F}}\left(N_{S}(P), N_{S}(\widehat{P})\right) \quad \text { and } \quad \psi^{\prime} \in \operatorname{Hom}_{\mathcal{F}}\left(N_{S}\left(P^{\prime}\right), N_{S}(\widehat{P})\right)
$$

such that $\psi(P)=\psi^{\prime}\left(P^{\prime}\right)=\widehat{P}$. Set $\widetilde{\varphi}=\left(\left.\psi^{\prime}\right|_{P^{\prime}}\right) \circ \varphi_{\circ}\left(\left.\psi\right|_{P}\right)^{-1} \in \operatorname{Aut}_{\mathcal{F}}(\widehat{P})$.

For each $x \in N_{\varphi}$, there exists $y \in N_{S}\left(P^{\prime}\right)$ such that $\varphi c_{x} \varphi^{-1}=c_{y}$ as elements of $\operatorname{Aut}\left(P^{\prime}\right)$. Then as automorphisms of $\widehat{P}, \widetilde{\varphi} c_{\psi(x)} \widetilde{\varphi}^{-1}=c_{\psi^{\prime}(y)}$. This shows that $\psi\left(N_{\varphi}\right) \leq N_{\widetilde{\varphi}}$. By $(\mathrm{IIB})_{\mathcal{H}}, \widetilde{\varphi}$ extends to a morphism $\widehat{\varphi} \in \operatorname{Hom}_{\mathcal{F}}\left(N_{\widetilde{\varphi}}, N_{S}(\widehat{P})\right)$.

Now fix $x \in N_{\varphi}$, and let $y \in N_{S}\left(P^{\prime}\right)$ be such that $\varphi c_{x} \varphi^{-1}=c_{y}$ as elements of $\operatorname{Aut}\left(P^{\prime}\right)$. The elements $\widehat{\varphi} \psi(x), \psi^{\prime}(y) \in N_{S}(\widehat{P})$ induce the same conjugation action on $\widehat{P}$, and thus differ by an element in $C_{S}(\widehat{P})$. Also, since $P^{\prime}$ is fully centralized, $\psi^{\prime}\left(C_{S}\left(P^{\prime}\right)\right)=C_{S}(\widehat{P})$, and hence

$$
\widehat{\varphi} \psi(x) \in \psi^{\prime}(y) \cdot C_{S}(\widehat{P})=\psi^{\prime}\left(y \cdot C_{S}\left(P^{\prime}\right)\right) \leq \psi^{\prime}\left(N_{S}\left(P^{\prime}\right)\right) .
$$

Thus $\widehat{\varphi} \psi\left(N_{\varphi}\right) \leq \psi^{\prime}\left(N_{S}\left(P^{\prime}\right)\right)$, and so $\widehat{\varphi} \circ \psi$ factors through some morphism $\bar{\varphi} \in$ $\operatorname{Hom}_{\mathcal{F}}\left(N_{\varphi}, N_{S}\left(P^{\prime}\right)\right)$ which extends $\varphi$. This finishes the proof of condition (II) $)_{\mathcal{H}}$.

As an immediate consequence of Lemma 2.3 we obtain the following alternative characterization of the conditions of saturation: a fusion system $\mathcal{F}$ over $S$ is saturated if and only if it satisfies the conditions $\left(\mathrm{I}^{\prime}\right)_{\mathcal{H}},(\mathrm{IIA})_{\mathcal{H}}$ and $(\mathrm{IIB})_{\mathcal{H}}$ where $\mathcal{H}$ is the set of all subgroups $P \leq S$.

Notation. Following the notation introduced in Lemma 2.3 for the conditions stated there, we also write $(-)_{Q}$ or $(-)_{>Q}$ for $(-)_{\mathcal{H}}$ when $\mathcal{H}=\{Q\}$ or $\mathcal{H}=\{P \mid Q \supsetneqq$ $P \leq S\}$, respectively. Given a fusion system $\mathcal{F}$ over $S$, let $\mathcal{S}$ be the set of all subgroups of $S$. For $P \leq S$, let $\mathcal{S}_{\geq P} \supseteq \mathcal{S}_{>P}$ be the sets of subgroups of $S$ which contain, or strictly contain, $P$.

We will now prove two lemmas which allow us to prove Theorem 2.2 by induction on the number of $\mathcal{F}$-conjugacy classes of subgroups of $S$ not in $\mathcal{H}$. 
Lemma 2.4. Let $\mathcal{F}$ be a fusion system over a finite $p$-group $S$, and let $\mathcal{H}$ be a set of subgroups of $S$ closed under $\mathcal{F}$-conjugacy. Let $\mathcal{P}$ be an $\mathcal{F}$-conjugacy class of subgroups of $S$ which is maximal among those not in $\mathcal{H}$. Assume $\mathcal{F}$ is $\mathcal{H}$-generated and $\mathcal{H}$-saturated. Then the following hold for any $P \in \mathcal{P}$ which is fully normalized in $\mathcal{F}$ :

(a) $N_{\mathcal{F}}(P)$ is $\mathcal{S}_{>P}$-saturated.

(b) Each $\varphi \in \operatorname{Aut}_{\mathcal{F}}(P)$ is a composite of restrictions of morphisms in $N_{\mathcal{F}}(P)$ between subgroups strictly containing $P$.

(c) $\mathcal{F}$ is $(\mathcal{H} \cup \mathcal{P})$-saturated if $N_{\mathcal{F}}(P)$ is $\mathcal{S}_{\geq P}$-saturated.

Proof. $\quad$ By a proper $\mathcal{P}$-pair will be meant a pair $(Q, P)$, where $P \supsetneqq Q \leq N_{S}(P)$ and $P \in \mathcal{P}$. Two proper $\mathcal{P}$-pairs $(Q, P)$ and $\left(Q^{\prime}, P^{\prime}\right)$ will be called $\mathcal{F}$-conjugate if there is an isomorphism $\varphi \in \operatorname{Iso}_{\mathcal{F}}\left(Q, Q^{\prime}\right)$ such that $\varphi(P)=P^{\prime}$. A proper $\mathcal{P}$-pair $(Q, P)$ will be called fully normalized if $\left|N_{N_{S}(P)}(Q)\right| \geq\left|N_{N_{S}\left(P^{\prime}\right)}\left(Q^{\prime}\right)\right|$ for all $\left(Q^{\prime}, P^{\prime}\right)$ in the same $\mathcal{F}$-conjugacy class.

The proof of the lemma is based on the following statements, whose proof will be carried out in Steps 1 to 4.

(1) If $(Q, P)$ is a fully normalized proper $\mathcal{P}$-pair, then $Q$ is fully centralized in $\mathcal{F}$ and

$$
\operatorname{Aut}_{N_{S}(P)}(Q) \in \operatorname{Syl}_{p}\left(\operatorname{Aut}_{N_{\mathcal{F}}(P)}(Q)\right) .
$$

(2) For each proper $\mathcal{P}$-pair $(Q, P)$, and each fully normalized proper $\mathcal{P}$-pair $\left(Q^{\prime}, P^{\prime}\right)$ which is $\mathcal{F}$-conjugate to $(Q, P)$, there is some morphism

$$
\psi \in \operatorname{Hom}_{\mathcal{F}}\left(N_{N_{S}(P)}(Q), N_{N_{S}\left(P^{\prime}\right)}\left(Q^{\prime}\right)\right)
$$

such that $\psi(P)=P^{\prime}$ and $\psi(Q)=Q^{\prime}$.

(3) There is a subgroup $\widehat{P} \in \mathcal{P}$ which is fully centralized in $\mathcal{F}$, and which has the property that for all $P \in \mathcal{P}$, there is a morphism $\varphi \in \operatorname{Hom}_{\mathcal{F}}\left(N_{S}(P), N_{S}(\widehat{P})\right)$ such that $\varphi(P)=\widehat{P}$.

(4) Let $(Q, P)$ be a proper $\mathcal{P}$-pair such that $P$ is fully normalized in $\mathcal{F}$. If $Q$ is fully normalized in $N_{\mathcal{F}}(P)$, then $(Q, P)$ is fully normalized. If $Q$ is fully centralized in $N_{\mathcal{F}}(P)$, then $Q$ is fully centralized in $\mathcal{F}$.

Note that point (3) implies that $\widehat{P}$ is fully normalized in $\mathcal{F}$, and that any other $P^{\prime} \in \mathcal{P}$ which is fully normalized in $\mathcal{F}$ has the same properties.

Assuming points (1)-(4) have been shown, the lemma is proven as follows:

(a) We show that conditions (I) and (II) hold in $N_{\mathcal{F}}(P)$ for all $Q \in \mathcal{S}_{>P}$. If $Q \supsetneqq P$ is fully normalized in $N_{\mathcal{F}}(P)$, then the proper $\mathcal{P}$-pair $(Q, P)$ is fully normalized by (4), and hence condition (I) holds in $N_{\mathcal{F}}(P)$ by (1). It remains to show condition (II). Also, by (4) again, if $P \supsetneqq Q \leq N_{S}(P)$ and $Q$ is fully centralized in $N_{\mathcal{F}}(P)$, then it is fully centralized in $\mathcal{F}$. Hence (II) holds automatically for morphisms $\varphi \in \operatorname{Hom}_{N_{\mathcal{F}}(P)}\left(Q, N_{S}(P)\right)$, since it holds in $\mathcal{F}$.

(b) Fix $\varphi \in \operatorname{Aut}_{\mathcal{F}}(P)$. Since $\mathcal{F}$ is $\mathcal{H}$-generated, there are subgroups

$$
P=P_{0}, P_{1}, \ldots, P_{k}=P \quad \text { in } \mathcal{P}, \quad \text { and } \quad P_{i} \supsetneqq Q_{i} \leq S, \quad Q_{i} \in \mathcal{H}
$$

and morphisms $\varphi_{i} \in \operatorname{Hom}_{\mathcal{F}}\left(Q_{i}, S\right)(0 \leq i \leq k-1)$, such that $\varphi_{i}\left(P_{i}\right)=P_{i+1}$ and 
$\varphi=\left.\left.\varphi_{k-1}\right|_{P_{k-1}} \circ \cdots \circ \varphi_{0}\right|_{P_{0}}$. Upon replacing each $Q_{i}$ by $N_{Q_{i}}\left(P_{i}\right) \supsetneqq P_{i}$, we can assume that $Q_{i} \leq N_{S}\left(P_{i}\right)$. By (3), there are morphisms $\chi_{i} \in \operatorname{Hom}_{\mathcal{F}}\left(N_{S}\left(P_{i}\right), N_{S}(P)\right)$ for each $i$ such that $\chi_{i}\left(P_{i}\right)=P$, where we take $\chi_{0}=\chi_{k}$ to be the identity. Upon replacing each $\varphi_{i}$ by $\chi_{i} \circ \varphi_{i} \circ \chi_{i}^{-1} \in \operatorname{Hom}_{\mathcal{F}}\left(\chi_{i}\left(Q_{i}\right), S\right)$, we can arrange that $P_{i}=P$ for all $i$. Thus $\varphi$ is a composite of restrictions of morphisms in $N_{\mathcal{F}}(P)$ between subgroups strictly containing $P$.

(c) Assume that $N_{\mathcal{F}}(P)$ is $\mathcal{S}_{\geq P}$-saturated. By Lemma 2.3 it is enough to check that Conditions $\left(\mathrm{I}^{\prime}\right)_{\mathcal{P}},(\mathrm{IIA})_{\mathcal{P}}$, and $(\mathrm{IIB})_{\mathcal{P}}$ are satisfied in $\mathcal{F}$. Condition $(\mathrm{IIA})_{\mathcal{P}}$ follows from point $(3)$. Since $\operatorname{Aut}_{\mathcal{F}}(P)=\operatorname{Aut}_{N_{\mathcal{F}}(P)}(P)$, it is clear that Condition $(\mathrm{IIB})_{\mathcal{P}}$ holds in $\mathcal{F}$. Finally, since $\operatorname{Aut}_{S}(P)=\operatorname{Aut}_{N_{S}(P)}(P)$, and since the properties of $\widehat{P}$ as described in point (3) hold for every fully normalized subgroup, $(\mathrm{I})_{\mathcal{P}}$ also holds, and this proves that $\mathcal{F}$ is $(\mathcal{H} \cup \mathcal{P})$-saturated.

In order to finish the proof, it remains to prove points (1)-(4).

Step 1: For any proper $\mathcal{P}$-pair $(Q, P)$, let $K_{P} \leq \operatorname{Aut}(Q)$ be defined by

$$
K_{P}=\{\varphi \in \operatorname{Aut}(Q) \mid \varphi(P)=P\} .
$$

If the pair $(Q, P)$ is fully normalized, then $Q$ is fully $K_{P}$-normalized in $\mathcal{F}$ in the sense of [5 Definition A.1]. Hence by [5. Proposition A.2(a)], $Q$ is fully centralized and

$$
\operatorname{Aut}_{N_{S}(P)}(Q)=\operatorname{Aut}_{S}(Q) \cap K_{P} \in \operatorname{Syl}_{p}\left(\operatorname{Aut}_{\mathcal{F}}(Q) \cap K_{P}\right)=\operatorname{Syl}_{p}\left(\operatorname{Aut}_{N_{\mathcal{F}}(P)}(Q)\right) .
$$

More precisely, this follows from the proof of 5 Proposition A.2], where we need only know that $\mathcal{F}$ satisfies the axioms of saturation on subgroups containing $Q$ and its $\mathcal{F}$-conjugates.

Step 2: Let $\left(Q^{\prime}, P^{\prime}\right)$ be any fully normalized proper $\mathcal{P}$-pair of subgroups of $S$ which is $\mathcal{F}$-conjugate to $(Q, P)$. Let $\varphi \in \operatorname{Iso}_{\mathcal{F}}\left(Q, Q^{\prime}\right)$ such that $\varphi(P)=P^{\prime}$. Since $\left(Q^{\prime}, P^{\prime}\right)$ is fully normalized, $Q^{\prime}$ is fully centralized and $\operatorname{Aut}_{N_{S}\left(P^{\prime}\right)}\left(Q^{\prime}\right) \in \operatorname{Syl}_{p}\left(\operatorname{Aut}_{N_{\mathcal{F}}\left(P^{\prime}\right)}\left(Q^{\prime}\right)\right)$ by (1).

Since $\varphi \operatorname{Aut}_{N_{S}(P)}(Q) \varphi^{-1}$ is a $p$-subgroup of $\operatorname{Aut}_{N_{\mathcal{F}}\left(P^{\prime}\right)}\left(Q^{\prime}\right)$, there is a morphism $\alpha \in \operatorname{Aut}_{N_{\mathcal{F}}\left(P^{\prime}\right)}\left(Q^{\prime}\right)$ such that

$$
\alpha \varphi \operatorname{Aut}_{N_{S}(P)}(Q) \varphi^{-1} \alpha^{-1} \leq \operatorname{Aut}_{N_{S}\left(P^{\prime}\right)}\left(Q^{\prime}\right) .
$$

Since $\mathcal{F}$ is $\mathcal{H}$-saturated, $\alpha \varphi$ extends to a morphism $\widetilde{\alpha \varphi} \in \operatorname{Hom}_{\mathcal{F}}\left(N_{\alpha \varphi}, S\right)$ by $(\mathrm{II})_{Q}$, where

$$
N_{\alpha \varphi}=\left\{x \in N_{S}(Q) \mid \alpha \varphi c_{x} \varphi^{-1} \alpha^{-1} \in \operatorname{Aut}_{S}\left(Q^{\prime}\right)\right\} \geq N_{N_{S}(P)}(Q) .
$$

Set $\psi=\left.\widetilde{\alpha \varphi}\right|_{N_{N_{S}(P)}(Q)} \in \operatorname{Hom}_{\mathcal{F}}\left(N_{N_{S}(P)}(Q), S\right)$. Then $\operatorname{Im}(\psi) \leq N_{N_{S}\left(P^{\prime}\right)}\left(Q^{\prime}\right)$ by construction. Moreover, $\left.\psi\right|_{Q}=\left.\alpha \varphi\right|_{Q},\left.\psi\right|_{P}=\left.\alpha \varphi\right|_{P}$, and hence $\psi(P)=P^{\prime}$ and $\psi(Q)=Q^{\prime}$.

Step 3: We first show, for any $P, P^{\prime} \in \mathcal{P}$, that there is a subgroup $P^{\prime \prime} \in \mathcal{P}$, and morphisms $\psi \in \operatorname{Hom}_{\mathcal{F}}\left(N_{S}(P), N_{S}\left(P^{\prime \prime}\right)\right)$ and $\psi^{\prime} \in \operatorname{Hom}_{\mathcal{F}}\left(N_{S}\left(P^{\prime}\right), N_{S}\left(P^{\prime \prime}\right)\right)$, such that $\psi(P)=P^{\prime \prime}=\psi^{\prime}\left(P^{\prime}\right)$.

Let $\mathcal{T}$ be the set of all sequences

$$
\xi=\left(P=P_{0}, Q_{0}, \varphi_{0} ; P_{1}, Q_{1}, \varphi_{1} ; \ldots ; P_{k-1}, Q_{k-1}, \varphi_{k-1} ; P_{k}=P^{\prime}\right)
$$

such that $P_{i} \supsetneqq Q_{i} \leq N_{S}\left(P_{i}\right), \varphi_{i} \in \operatorname{Hom}_{\mathcal{F}}\left(Q_{i}, N_{S}\left(P_{i+1}\right)\right)$, and $\varphi_{i}\left(P_{i}\right)=P_{i+1}$. Let $\mathcal{T}_{r} \subseteq \mathcal{T}$ be the subset of those $\xi$ for which there is no $1 \leq i \leq k-1$ such that $Q_{i}=N_{S}\left(P_{i}\right)=\varphi_{i-1}\left(Q_{i-1}\right)$. Let $\mathcal{T} \stackrel{R}{\longrightarrow} \mathcal{T}_{r}$ be the "reduction" map, which removes 
any $P_{i}$ such that $Q_{i}=N_{S}\left(P_{i}\right)=\varphi_{i-1}\left(Q_{i-1}\right)$ (and replaces $\varphi_{i-1}$ and $\varphi_{i}$ by their composite).

Define

$$
I(\xi)=\left\{0 \leq i \leq k-1 \mid Q_{i} \supsetneqq N_{S}\left(P_{i}\right) \text { and } \varphi_{i}\left(Q_{i}\right) \supsetneqq N_{S}\left(P_{i+1}\right)\right\} .
$$

If $\xi \in \mathcal{T}$ and $I(\xi) \neq \emptyset$, define

$$
\lambda(\xi)=\min _{i \in I(\xi)}\left[Q_{i}: P_{i}\right] \geq p .
$$

The main observation needed to prove point (3) is that there exists an element $\xi \in \mathcal{T}_{r}$ such that $I(\xi)=\emptyset$. Note first that $\mathcal{T} \neq \emptyset$, since $\mathcal{F}$ is $\mathcal{H}$-generated (and since $Q \supsetneqq P$ implies $N_{Q}(P) \supsetneqq P$ ). Hence (by the existence of the retraction functor $R$ ) $\mathcal{T}_{r} \neq \emptyset$.

Fix an element $\xi \in \mathcal{T}_{r}$ such that $I(\xi) \neq \emptyset$. We will construct $\widehat{\xi} \in \mathcal{T}_{r}$ such that either $I(\widehat{\xi})=\emptyset$, or $\lambda(\widehat{\xi})>\lambda(\xi)$. For each $i \in I(\xi)$, choose a fully normalized proper $\mathcal{P}$-pair $\left(Q_{i}^{\prime \prime}, P_{i}^{\prime \prime}\right)$ which is $\mathcal{F}$-conjugate to $\left(Q_{i}, P_{i}\right)$, and apply $(2)$ to choose homomorphisms

$$
\psi_{i} \in \operatorname{Hom}_{\mathcal{F}}\left(N_{N_{S}\left(P_{i}\right)}\left(Q_{i}\right), S\right) \quad \text { and } \quad \psi_{i}^{\prime} \in \operatorname{Hom}_{\mathcal{F}}\left(N_{N_{S}\left(P_{i+1}\right)}\left(\varphi_{i}\left(Q_{i}\right)\right), S\right)
$$

such that $\psi_{i}\left(P_{i}\right)=\psi_{i}^{\prime}\left(P_{i+1}\right)=P_{i}^{\prime \prime}$ and $\psi_{i}\left(Q_{i}\right)=\psi_{i}^{\prime}\left(Q_{i+1}\right)=Q_{i}^{\prime \prime}$. Set

$$
\widetilde{Q}_{i}=N_{N_{S}\left(P_{i}\right)}\left(Q_{i}\right) \supsetneqq Q_{i} \quad \text { and } \quad \widetilde{Q}_{i}^{\prime}=\psi_{i}^{\prime}\left(N_{N_{S}\left(P_{i+1}\right)}\left(\varphi_{i}\left(Q_{i}\right)\right)\right) \supsetneqq \psi_{i}^{\prime}\left(Q_{i}\right) .
$$

Note that if $(Q, P)$ is a proper $\mathcal{P}$-pair with $P \supsetneqq Q \supsetneqq N_{S}(P)$, then $N_{N_{S}(P)}(Q) \supsetneqq Q$. Thus upon replacing the sequence $\left(P_{i}, Q_{i}, \varphi_{i} ; P_{i+1}\right)$ in $\xi$ by

$$
\left(P_{i}, \widetilde{Q}_{i}, \psi_{i} ; P_{i}^{\prime \prime}, \widetilde{Q}_{i}^{\prime},\left(\psi_{i}^{\prime}\right)^{-1} ; P_{i+1}\right)
$$

and similarly for the other components of $I(\xi)$, we obtain a new element $\xi^{\prime} \in \mathcal{T}$, such that either $I\left(\xi^{\prime}\right)=\emptyset$ or $\lambda\left(\xi^{\prime}\right)>\lambda(\xi)$ (by construction $\left[\widetilde{Q}_{i}: P_{i}\right]>\left[Q_{i}: P_{i}\right]$ and $\left.\left[\widetilde{Q}_{i}^{\prime}: P^{\prime}\right]>\left[Q_{i}: P_{i}\right]\right)$. Then $\widehat{\xi}=R\left(\xi^{\prime}\right) \in \mathcal{T}_{r}$ is also such that either $I(\widehat{\xi})=\emptyset$ or $\lambda(\widehat{\xi})>\lambda(\xi)$.

Since the function $\lambda$ is bounded above, it follows by induction that there is $\xi \in \mathcal{T}_{r}$ such that $I(\xi)=\emptyset$. Write

$$
\xi=\left(P_{0}, Q_{0}, \varphi_{0} ; \ldots ; P_{k-1}, Q_{k-1}, \varphi_{k-1} ; P_{k}\right) \in \mathcal{T}_{r} \quad\left(P_{0}=P, P_{k}=P^{\prime}\right) .
$$

The assumption $I(\xi)=\emptyset$ implies that for each $i$, either $Q_{i}=N_{S}\left(P_{i}\right)$ (hence $\left.\left|N_{S}\left(P_{i}\right)\right| \leq\left|N_{S}\left(P_{i+1}\right)\right|\right)$, or $\varphi_{i}\left(Q_{i}\right)=N_{S}\left(P_{i+1}\right)$ (hence $\left|N_{S}\left(P_{i}\right)\right| \geq\left|N_{S}\left(P_{i+1}\right)\right|$ ).

Thus when $\xi \in \mathcal{T}_{r}$, there is no $1 \leq i \leq k-1$ such that $\left|N_{S}\left(P_{i}\right)\right|<\left|N_{S}\left(P_{i-1}\right)\right|$ and also $\left|N_{S}\left(P_{i}\right)\right|<\left|N_{S}\left(P_{i+1}\right)\right|$. So if we choose $0 \leq j \leq k$ such that $\left|N_{S}\left(P_{j}\right)\right|$ is maximal, then

$$
\left|N_{S}(P)\right| \leq\left|N_{S}\left(P_{1}\right)\right| \leq\left|N_{S}\left(P_{2}\right)\right| \leq \cdots \leq\left|N_{S}\left(P_{j}\right)\right|
$$

and

$$
\left|N_{S}\left(P_{j}\right)\right| \geq\left|N_{S}\left(P_{j-1}\right)\right| \geq \cdots \geq\left|N_{S}\left(P_{k-1}\right)\right| \geq\left|N_{S}\left(P^{\prime}\right)\right| .
$$

Since $I(\xi)=\emptyset$, this implies that $Q_{i}=N_{S}\left(P_{i}\right)$ for all $i<j$, and that $\varphi_{i}\left(Q_{i}\right)=$ $N_{S}\left(P_{i+1}\right)$ for all $j \leq i \leq k-1$. So upon setting $P^{\prime \prime}=P_{j}$, we obtain homomorphisms

$$
\psi=\varphi_{j-1} \circ \cdots \circ \varphi_{0} \in \operatorname{Hom}_{\mathcal{F}}\left(N_{S}(P), N_{S}\left(P^{\prime \prime}\right)\right)
$$

and

$$
\psi^{\prime}=\left(\varphi_{k-1} \circ \cdots \circ \varphi_{j}\right)^{-1} \in \operatorname{Hom}_{\mathcal{F}}\left(N_{S}\left(P^{\prime}\right), N_{S}\left(P^{\prime \prime}\right)\right)
$$


such that $\psi(P)=P^{\prime \prime}=\psi^{\prime}\left(P^{\prime}\right)$.

This was shown for an arbitrary pair of subgroups $P, P^{\prime} \in \mathcal{P}$. By successively applying the above construction to the subgroups in the $\mathcal{F}$-conjugacy class $\mathcal{P}$, it now follows easily that there is some $\widehat{P} \in \mathcal{P}$ such that for all $P \in \mathcal{P}$, there is a morphism $\varphi \in \operatorname{Hom}_{\mathcal{F}}\left(N_{S}(P), N_{S}(\widehat{P})\right)$ such that $\varphi(P)=\widehat{P}$. Note that $\widehat{P}$ is fully normalized since $N_{S}(\widehat{P})$ contains an injective image of any other $N_{S}(P)$ for $P \in \mathcal{P}$. For the same reason, $\widehat{P}$ is fully centralized in $\mathcal{F}$ : its centralizer contains an injective image of the centralizer of any other subgroup in the $\mathcal{F}$-conjugacy class $\mathcal{P}$.

Step 4: Fix a proper $\mathcal{P}$-pair $(Q, P)$ such that $P$ is fully normalized in $\mathcal{F}$. By $(3)$, the pair $\left(N_{S}(P), P\right)$ is $\mathcal{F}$-conjugate to $\left(N_{S}(\widehat{P}), \widehat{P}\right)$. Hence for every $P^{\prime} \in \mathcal{P}$, there is

$$
\psi \in \operatorname{Hom}_{\mathcal{F}}\left(N_{S}\left(P^{\prime}\right), N_{S}(P)\right)
$$

such that $\psi\left(P^{\prime}\right)=P$.

Assume $Q$ is fully normalized in $N_{\mathcal{F}}(P)$. Let $\left(Q^{\prime}, P^{\prime}\right)$ be any proper $\mathcal{P}$-pair $\mathcal{F}$-conjugate to $(Q, P)$, and choose $\psi$ as above. Set $Q^{\prime \prime}=\psi\left(Q^{\prime}\right)$. Then $\psi$ sends $N_{N_{S}\left(P^{\prime}\right)}\left(Q^{\prime}\right)$ injectively into $N_{N_{S}(P)}\left(Q^{\prime \prime}\right)$. So

$$
\left|N_{N_{S}\left(P^{\prime}\right)}\left(Q^{\prime}\right)\right| \leq\left|N_{N_{S}(P)}\left(Q^{\prime \prime}\right)\right| \leq\left|N_{N_{S}(P)}(Q)\right| ;
$$

where the last inequality holds since $Q$ is fully normalized in $N_{\mathcal{F}}(P)$. This shows that the pair $(P, Q)$ is fully normalized.

Finally, assume $Q$ is fully centralized in $N_{\mathcal{F}}(P)$, and let $Q^{\prime}$ be any other subgroup in the $\mathcal{F}$-conjugacy class of $Q$. Fix $\varphi \in \operatorname{Iso}_{\mathcal{F}}\left(Q, Q^{\prime}\right)$, and set $P^{\prime}=\varphi(P)$. Again, choose $\psi$ as above, and set $Q^{\prime \prime}=\psi\left(Q^{\prime}\right)$. Then $\left|C_{S}\left(Q^{\prime}\right)\right| \leq\left|C_{S}\left(Q^{\prime \prime}\right)\right|$ since $\psi$ sends the first subgroup injectively into the second, and $\left|C_{S}\left(Q^{\prime \prime}\right)\right| \leq\left|C_{S}(Q)\right|$ since $Q$ is fully centralized in $N_{\mathcal{F}}(P)$ and the pairs $(Q, P)$ and $\left(Q^{\prime \prime}, P\right)$ are $\mathcal{F}$-conjugate. This shows that $Q$ is fully centralized in $\mathcal{F}$.

Lemma 2.4 reduces the problem of proving $\mathcal{P}$-saturation, for an $\mathcal{F}$-conjugacy class $\mathcal{P}$, to the case where $\mathcal{P}=\{P\}$ and $P$ is normal in $\mathcal{F}$. This case is handled in the next lemma.

Lemma 2.5. Let $\mathcal{F}$ be a fusion system over a p-group $S$. Assume that $P \triangleleft S$ is normal in $\mathcal{F}$, and that $\mathcal{F}$ is $\mathcal{S}_{>P}$-generated and $\mathcal{S}_{>P}$-saturated. Assume furthermore that either $P$ is not $\mathcal{F}$-centric, or $\operatorname{Out}_{S}(P) \cap O_{p}\left(\operatorname{Out}_{\mathcal{F}}(P)\right) \neq 1$. Then $\mathcal{F}$ is $\mathcal{S}_{\geq P^{-}}$ saturated.

Proof. Define

$$
P^{*}=\left\{x \in S \mid c_{x} \in O_{p}\left(\operatorname{Aut}_{\mathcal{F}}(P)\right)\right\} .
$$

It follows from the definition that $P^{*} \triangleleft S$, and we claim that $P^{*}$ is strongly closed in $\mathcal{F}$. Assume that $x \in P^{*}$ is $\mathcal{F}$-conjugate to $y \in S$. Since $P$ is normal in $\mathcal{F}$, there exists $\psi \in \operatorname{Hom}_{\mathcal{F}}(\langle x, P\rangle,\langle y, P\rangle)$ which satisfies $\psi(P)=P$ and $\psi(x)=y$. In particular, $\psi \circ c_{x} \circ \psi^{-1}=c_{y}$. It follows that $y \in P^{*}$, since $c_{x} \in O_{p}\left(\operatorname{Aut}_{\mathcal{F}}(P)\right)$.

Note also that $P^{*} \geq P \cdot C_{S}(P)$. So by the assumption $\operatorname{Out}_{S}(P) \cap O_{p}\left(\operatorname{Out}_{\mathcal{F}}(P)\right) \neq 1$ if $P$ is $\mathcal{F}$-centric, or by definition if $P$ is not $\mathcal{F}$-centric, $P \supsetneqq P^{*}$ in all cases.

Since $\mathcal{F}$ is assumed to be $\mathcal{S}_{>P}$-saturated, we need only to prove conditions $(\mathrm{I})_{P}$ and $(\mathrm{II})_{P}$. We first prove that these conditions follow from the following statement: 
$(* *)$ each $\varphi \in \operatorname{Aut}_{\mathcal{F}}(P)$ extends to some $\bar{\varphi} \in \operatorname{Aut}_{\mathcal{F}}\left(P^{*}\right)$.

Since $P$ is normal in $\mathcal{F}$, it is the only subgroup in its $\mathcal{F}$-conjugacy class, and hence it is fully centralized and fully normalized. It is also clear that $P^{*}$ is fully normalized in $\mathcal{F}$, since $P^{*} \triangleleft S$. Hence $\operatorname{Aut}_{S}\left(P^{*}\right) \in \operatorname{Syl}_{p}\left(\operatorname{Aut}_{\mathcal{F}}\left(P^{*}\right)\right)$ by $(\mathrm{I})_{>P}$. The restriction map from $\operatorname{Aut}_{\mathcal{F}}\left(P^{*}\right)$ to $\operatorname{Aut}_{\mathcal{F}}(P)$ is surjective by $(* *)$, and $\operatorname{so~} \operatorname{Aut}_{S}(P) \in \operatorname{Syl}_{p}\left(\operatorname{Aut}_{\mathcal{F}}(P)\right)$. Therefore condition $(\mathrm{I})_{P}$ holds.

Next we prove condition $(\mathrm{II})_{P}$ : that each automorphism $\varphi \in \operatorname{Aut}_{\mathcal{F}}(P)$ extends to a morphism defined on $N_{\varphi}$. By $(* *), \varphi$ extends to some $\psi \in \operatorname{Aut}_{\mathcal{F}}\left(P^{*}\right)$. Consider the groups of automorphisms

$$
\begin{aligned}
K & =\left\{\chi \in \operatorname{Aut}_{S}\left(P^{*}\right)|\chi|_{P}=c_{x} \text { some } x \in N_{\varphi}\right\} \\
K_{0} & =\left\{\chi \in \operatorname{Aut}_{\mathcal{F}}\left(P^{*}\right)|\chi|_{P}=\operatorname{Id}_{P}\right\} \triangleleft \operatorname{Aut}_{\mathcal{F}}\left(P^{*}\right) .
\end{aligned}
$$

By definition, for all $x \in N_{\varphi}$, we have $\left.\left(\psi c_{x} \psi^{-1}\right)\right|_{P}=\left.\chi\right|_{P}$ for some $\chi \in \operatorname{Aut}_{S}\left(P^{*}\right)$. In other words, as subgroups of $\operatorname{Aut}\left(P^{*}\right)$,

$$
\psi K \psi^{-1} \leq\left\{\psi c_{x} \psi^{-1} \mid x \in N_{\varphi}\right\} \cdot\left(\psi K_{0} \psi^{-1}\right) \leq \operatorname{Aut}_{S}\left(P^{*}\right) \cdot\left(\psi K_{0} \psi^{-1}\right) .
$$

In general, if $S \in \operatorname{Syl}_{p}(G), H \triangleleft G$, and $P \leq S H$ is a $p$-subgroup, then there is $x \in H$ such that $P \leq x S x^{-1}$. Applied to this situation (with $G=\operatorname{Aut}_{\mathcal{F}}\left(P^{*}\right)$, $S=\operatorname{Aut}_{S}\left(P^{*}\right), H=\psi K_{0} \psi^{-1}$, and $\left.P=\psi K \psi^{-1}\right)$, we see that there is $\chi \in K_{0}$ such that

$$
(\psi \chi) K(\psi \chi)^{-1}=\left(\psi \chi \psi^{-1}\right)\left(\psi K \psi^{-1}\right)\left(\psi \chi \psi^{-1}\right)^{-1} \leq \operatorname{Aut}_{S}\left(P^{*}\right) .
$$

Also, $P^{*}$ is fully centralized in $\mathcal{F}$ by $(\mathrm{I})_{>P}$, since $P^{*}$ is fully normalized. So by $(\mathrm{II})_{>P}, \psi \chi \in \operatorname{Aut}_{\mathcal{F}}\left(P^{*}\right)$ extends to a morphism $\bar{\varphi}$ defined on $N_{S}^{K}\left(P^{*}\right) \geq N_{\varphi}$, and $\left.\bar{\varphi}\right|_{P}=\left.\psi\right|_{P}=\varphi$ since $\left.\chi\right|_{P}=\operatorname{Id}_{P}$.

In order to finish the proof, it remains to prove $(* *)$. Since any $\varphi \in \operatorname{Aut}_{\mathcal{F}}(P)$ is a composite of automorphisms of $P$ which extend to strictly larger subgroups, it suffices to show $(* *)$ when $\varphi$ itself extends to $\widetilde{\varphi} \in \operatorname{Iso}_{\mathcal{F}}\left(Q_{1}, Q_{2}\right)$, where $Q_{i} \supsetneqq P$. Note that

$$
\widetilde{\varphi}\left(Q_{1} \cap P^{*}\right)=Q_{2} \cap P^{*}
$$

since $P^{*}$ is strongly closed in $\mathcal{F}$.

We show $(* *)$ by induction on the index $\left[P^{*}: P^{*} \cap Q_{1}\right]=\left[P^{*}: P^{*} \cap Q_{2}\right]$. If this index is 1, i.e., if $Q_{1} \geq P^{*}$, then $\widetilde{\varphi}\left(P^{*}\right)=P^{*}$ by $(1)$, and hence $\left.\bar{\varphi} \stackrel{\text { def }}{=} \widetilde{\varphi}\right|_{P^{*}}$ lies in $\operatorname{Aut}_{\mathcal{F}}\left(P^{*}\right)$ and extends $\varphi$.

Now assume $Q_{1} \nsucceq P^{*}$, let $Q_{3}$ be any subgroup $\mathcal{F}$-conjugate to $Q_{1}$ and $Q_{2}$ and fully normalized in $\mathcal{F}$, and fix $\varphi \in \operatorname{Iso}_{\mathcal{F}}\left(Q_{2}, Q_{3}\right)$. Upon replacing $\widetilde{\varphi}$ by $\psi$ and by $\psi \circ \widetilde{\varphi}$, we are reduced to proving the result when the target group is fully normalized. So assume $Q_{2}$ is fully normalized (and hence, by $(\mathrm{I})_{>P}$, fully centralized).

This time, consider the groups of automorphisms

$$
\begin{aligned}
K & =\left\{\chi \in \operatorname{Aut}_{\mathcal{F}}\left(Q_{2}\right)|\chi|_{P} \in O_{p}\left(\operatorname{Aut}_{\mathcal{F}}(P)\right)\right\} \\
K_{0} & =\left\{\chi \in \operatorname{Aut}_{\mathcal{F}}\left(Q_{2}\right)|\chi|_{P}=\operatorname{Id}_{P}\right\} .
\end{aligned}
$$

Both $K$ and $K_{0}$ are normal subgroups of $\operatorname{Aut}_{\mathcal{F}}\left(Q_{2}\right)$. Also, $K / K_{0}$ is a $p$-group, since there is a monomorphism $K / K_{0} \rightarrow O_{p}\left(\operatorname{Aut}_{\mathcal{F}}\left(Q_{2}\right)\right)$. So any two Sylow $p$-subgroups of $K$ are conjugate by an element of $K_{0}$.

By definition, $\operatorname{Aut}_{P^{*}}\left(Q_{1}\right)$ is a $p$-subgroup of $\operatorname{Aut}_{\mathcal{F}}\left(Q_{1}\right)$, all of whose elements restrict to elements of $O_{p}\left(\operatorname{Aut}_{\mathcal{F}}(P)\right)$. Hence $\widetilde{\varphi} \operatorname{Aut}_{P^{*}}\left(Q_{1}\right) \widetilde{\varphi}^{-1}$ is a $p$-subgroup of $K$. 
Since $Q_{2}$ is fully normalized, $\operatorname{Aut}_{S}\left(Q_{2}\right) \in \operatorname{Syl}_{p}\left(\operatorname{Aut}_{\mathcal{F}}\left(Q_{2}\right)\right)$, and hence $\operatorname{Aut}_{P^{*}}\left(Q_{2}\right)=$ $K \cap \operatorname{Aut}_{S}\left(Q_{2}\right)$ is a Sylow $p$-subgroup of $K$. Thus there is $\chi \in K_{0}$ such that

$$
\chi \widetilde{\varphi} \operatorname{Aut}_{P^{*}}\left(Q_{1}\right) \widetilde{\varphi}^{-1} \chi^{-1} \leq \operatorname{Aut}_{P^{*}}\left(Q_{2}\right) .
$$

In particular, $N_{P^{*} Q_{1}}\left(Q_{1}\right) \leq N_{\chi \widetilde{\varphi}}$. Since $Q_{2}$ is fully centralized, condition (II) $)_{>P}$ now implies that $\chi \widetilde{\varphi}$ extends to a morphism $\widetilde{\varphi}^{\prime} \in \operatorname{Hom}_{\mathcal{F}}\left(Q_{1}^{\prime}, N_{S}\left(Q_{2}\right)\right)$, where $Q_{1}^{\prime}=$ $N_{P^{*} Q_{1}}\left(Q_{1}\right)$. Furthermore, $\left.\widetilde{\varphi}^{\prime}\right|_{P}=\left.\widetilde{\varphi}\right|_{P}$ since $\chi \in K_{0}$.

By assumption, $P^{*} Q_{1} \supsetneqq Q_{1}$, and so $Q_{1}^{\prime}=N_{P^{*} Q_{1}}\left(Q_{1}\right) \supsetneqq Q_{1}$. Also, $Q_{1}^{\prime}$ is generated by $Q_{1}$ and $Q_{1}^{\prime} \cap P^{*}$ since $Q_{1} \leq Q_{1}^{\prime} \leq P^{*} Q_{1}$. Hence $Q_{1}^{\prime} \cap P^{*} \supsetneqq Q_{1} \cap P^{*}$. This shows that

$$
\left[P^{*}: P^{*} \cap Q_{1}^{\prime}\right]<\left[P^{*}: P^{*} \cap Q_{1}\right]
$$

and so $(* *)$ now follows by the induction hypothesis.

We are now ready to prove Theorem 2.2

Proof Proof of Theorem 2.2. We are given a set $\mathcal{H}$ of subsets of $S$, closed under $\mathcal{F}$-conjugacy, such that $\mathcal{F}$ is $\mathcal{H}$-generated and $\mathcal{H}$-saturated, and such that condition

$(*)$ each $\mathcal{F}$-conjugacy class of subgroups of $S$ which are $\mathcal{F}$-centric but not in $\mathcal{H}$ contains at least one subgroup $P$ such that $\operatorname{Out}_{S}(P) \cap O_{p}\left(\operatorname{Out}_{\mathcal{F}}(P)\right) \neq 1$.

holds. We will prove, by induction on the number of $\mathcal{F}$-conjugacy classes of subgroups of $S$ not in $\mathcal{H}$, that $\mathcal{F}$ is saturated. If $\mathcal{H}$ contains all subgroups, then we are done. Otherwise, let $\mathcal{P}$ be any $\mathcal{F}$-conjugacy class of subgroups of $S$ which is maximal among those not in $\mathcal{H}$. We will show that $\mathcal{F}$ is also $(\mathcal{H} \cup \mathcal{P})$-saturated. Since $\mathcal{F}$ is clearly $(\mathcal{H} \cup \mathcal{P})$-generated, the result then follows by the induction hypothesis.

By Lemma 2.4 for any fully normalized subgroup $P \in \mathcal{P}$, the normalizer fusion system $N_{\mathcal{F}}(P)$ is $\mathcal{S}_{>P}$-saturated, and $\operatorname{Aut}_{\mathcal{F}}(P)$ is generated by restrictions of morphisms in $N_{\mathcal{F}}(P)$ between subgroups of $N_{S}(P)$ which strictly contain $P$.

Let $\mathcal{F}_{0}$ be the fusion system over $S_{0} \stackrel{\text { def }}{=} N_{S}(P)$ generated by the restriction of $N_{\mathcal{F}}(P)$ to $\mathcal{S}_{>P}$, that is, the smallest fusion system over $S_{0}$ for which morphisms between subgroups in $\mathcal{S}_{>P}$ are the same as those in $N_{\mathcal{F}}(P)$. Then $\operatorname{Aut}_{\mathcal{F}_{0}}(P)=$ $\operatorname{Aut}_{\mathcal{F}}(P)$, and $\mathcal{F}_{0}$ is $\mathcal{S}_{>}$-saturated and $\mathcal{S}_{>P}$-generated. Also, by the assumption $(*)$, either $P$ is not centric in $\mathcal{F}$ (hence not centric in $\mathcal{F}_{0}$ ), or $\operatorname{Out}_{S}(P) \cap O_{p}\left(\operatorname{Out}_{\mathcal{F}_{0}}(P)\right) \neq$ 1. Then $\mathcal{F}_{0}$ is $\mathcal{S}_{\geq P}$-saturated by Lemma $[2.5$ and so $\mathcal{F}$ is $(\mathcal{H} \cup \mathcal{P})$-saturated by Lemma 2.4 again.

We end this section with a description of a example which shows why the assumption $(*)$ in Theorem 2.2 $\left(\operatorname{Out}_{S}(P) \cap O_{p}\left(\operatorname{Out}_{\mathcal{F}}(P)\right) \neq 1\right.$ if $P$ is not centric) is needed. We use the following standard notation: if $k$ is a finite field, and $n \geq 1$, then $\Sigma L_{n}(k)$ denotes the semidirect product of $S L_{n}(k)$ with the group of field automorphisms of $k$. This group has an obvious action on the vector space $k^{n}$ and on the projective space $\mathbb{P}\left(k^{n}\right)$. It is not hard to see that $\Sigma L_{2}\left(\mathbb{F}_{4}\right) \cong S_{5}$ : via its permutation action on the five points in $\mathbb{P}\left(\mathbb{F}_{4}{ }^{2}\right)$.

Let $\Gamma=\mathbb{F}_{4}^{2} \rtimes S_{5}$, where $S_{5}$ acts on $\mathbb{F}_{4}^{2}$ via the above isomorphism. Note that $\Gamma$ can be identified with the subgroup of $\Sigma L_{3}\left(\mathbb{F}_{4}\right)$ generated by matrices with bottom row $(0,0,1)$ and the field automorphism. Therefore $\Gamma$ acts faithfully on $P=\mathbb{F}_{4}^{3}$. 
We will define a fusion system $\mathcal{F}$ over $S=P \rtimes S^{\prime}$, where $S^{\prime}=\langle(12),(45)\rangle \supsetneqq$ $S_{5} \leq \Gamma$. Consider the following subgroups of $S: Q_{1}=P \rtimes\langle(12)\rangle, Q_{2}=P \rtimes\langle(45)\rangle$, and $Q_{3}=P \rtimes\langle(12)(45)\rangle$. We regard all of these groups, including $\Gamma$, as subgroups of $P \rtimes \Gamma$.

To define the morphisms in the fusion system $\mathcal{F}$, let $x \in O_{2}(\Gamma) \cong \mathbb{F}_{4}^{2}$ be the element of order two which centralizes $S^{\prime}$, and consider the subgroups $R_{1}=$ $\left\langle S^{\prime},(345)\right\rangle, R_{2}=\left\langle S^{\prime},(123)\right\rangle$, and $R_{2}^{\prime}=x R_{2} x^{-1}$. $\operatorname{Set}_{\operatorname{Out}_{\mathcal{F}}}(S)=1, \operatorname{Aut}_{\mathcal{F}}\left(Q_{1}\right)=$ $\operatorname{Aut}_{P R_{1}}\left(Q_{1}\right), \operatorname{Aut}_{\mathcal{F}}\left(Q_{2}\right)=\operatorname{Aut}_{P R_{2}^{\prime}}\left(Q_{2}\right)$, and $\operatorname{Aut}_{\mathcal{F}}\left(Q_{3}\right)=\operatorname{Aut}_{S}\left(Q_{3}\right)$. All other morphisms in the fusion system are restrictions of the ones just described. Note in particular that $\operatorname{Out}_{\mathcal{F}}\left(Q_{1}\right) \cong S_{3}, \operatorname{Out}_{\mathcal{F}}\left(Q_{2}\right) \cong S_{3}$, and $\operatorname{Aut}_{\mathcal{F}}(P)=\left\langle R_{1}, R_{2}^{\prime}\right\rangle=\Gamma$. The last equality holds since $\left\langle P, R_{1}, R_{2}^{\prime}\right\rangle / P=\left\langle S^{\prime},(123),(345)\right\rangle=S_{5}$; and $\left\langle R_{1}, R_{2}^{\prime}\right\rangle$ cannot be a splitting of $\Gamma / P$ in $\Gamma$ since any splitting containing $S^{\prime}$ must be $P$ conjugate to the given $S_{5} \leq \Gamma$; so $\left\langle R_{1}, R_{2}^{\prime}\right\rangle \cap P \neq 1$, and $\left\langle R_{1}, R_{2}^{\prime}\right\rangle \geq P$ since $P$ is irreducible as an $S_{5}$-representation.

Consider the set of subgroups $\mathcal{H}=\left\{S, Q_{1}, Q_{2}, Q_{3}\right\}$. It follows from the above description of morphisms in $\mathcal{F}$ that the subgroups in $\mathcal{H}$ are the only $\mathcal{F}$-centric, $\mathcal{F}$-radical subgroups. Also, $\mathcal{F}$ is $\mathcal{H}$-generated by construction, and one can check that $\mathcal{F}$ is $\mathcal{H}$-saturated. But $\mathcal{F}$ is not saturated, since axiom $(\mathrm{I})_{P}$ fails: $\operatorname{Aut}_{S}(P) \notin$ $\operatorname{Syl}_{2}\left(\operatorname{Aut}_{\mathcal{F}}(P)\right)$ since $\operatorname{Aut}_{S}(P) \cong C_{2}^{2}$ and $\operatorname{Aut}_{\mathcal{F}}(P) \cong \Gamma$. (One can also show that $(\mathrm{II})_{P}$ fails.) Note that $\operatorname{Out}_{S}(P) \cap O_{2}\left(\operatorname{Out}_{\mathcal{F}}(P)\right)=S^{\prime} \cap O_{2}(\Gamma)=1$, so Condition $(*)$ in Theorem 2.2 does not hold.

\section{Expanding and restricting the classifying space: quasicentric subgroups}

The goal of this section is to show how the centric linking system of a $p$-local finite group $(S, \mathcal{F}, \mathcal{L})$ can be extended to a larger category or restricted to a smaller one without changing the homotopy type of the nerve of $\mathcal{L}$.

One motivation for doing this is a problem which frequently occurs when trying to construct maps between $p$-local finite groups. A functor between fusion systems need not send centric subgroups to centric subgroups, in which case it cannot be lifted to a functor between associated centric linking systems. One could try to get around this by extending the linking systems to include all subgroups as objects. There is in fact a natural extension of the linking system to a category whose objects are all subgroups of $S$, but in general the homotopy type of the $p$-completed nerve is not preserved by this extension.

We introduce here the collection of $\mathcal{F}$-quasicentric subgroups, which contains the centric subgroups and supports an associated linking system $\mathcal{L}^{q}$ with properties analogous to those of the centric one. The important fact proved in this section is that the nerve of $\mathcal{L}^{q}$ is homotopy equivalent to $|\mathcal{L}|$. Moreover, any full subcategory of $\mathcal{L}^{q}$ whose object set contains all subgroups which are centric and radical also has nerve homotopy equivalent to $|\mathcal{L}|$.

DeFinition 3.1. Let $\mathcal{F}$ be a saturated fusion system over a p-group $S$. A subgroup $P \leq S$ is called $\mathcal{F}$-quasicentric if for each $P^{\prime}$ which is fully centralized in $\mathcal{F}$ and $\mathcal{F}$-conjugate to $P$, the centralizer system $C_{\mathcal{F}}\left(P^{\prime}\right)$ is the fusion system of the 
p-group $C_{S}\left(P^{\prime}\right)$. We let $\mathcal{F}^{q} \subseteq \mathcal{F}$ denote the full subcategory whose objects are the $\mathcal{F}$-quasicentric subgroups of $S$.

The simplest examples where $\mathcal{F}$-quasicentric subgroups need not be $\mathcal{F}$-centric are those where $\mathcal{F}=\mathcal{F}_{S}(S)$ is the fusion system of a $p$-group $S$ : every subgroup of $S$ is $\mathcal{F}$-quasicentric (while the trivial subgroup, at least, is not $\mathcal{F}$-centric). A more interesting example is given by considering a finite group $G$ with a normal subgroup $H$ of $p$-power index. Fix $S \in \operatorname{Syl}_{p}(G)$, and set $S_{0}=S \cap H \in \operatorname{Syl}_{p}(H)$. It is not too hard to see that each $\mathcal{F}_{S_{0}}(H)$-quasicentric subgroup of $S_{0}$ is also $\mathcal{F}_{S}(G)$-quasicentric, something which is not true for centric subgroups (consider, for instance, the case $A_{6} \triangleleft \Sigma_{6}$ ). It was this last observation which initially led us to consider this class of subgroups.

When $\mathcal{F}$ is a saturated fusion system over $S$, a subgroup $P \leq S$ is $\mathcal{F}$-quasicentric if and only if there is no $P^{\prime}$ which is $\mathcal{F}$-conjugate to $P$, and no $Q \leq C_{S}\left(P^{\prime}\right) \leq S$ and $\alpha \in \operatorname{Aut}_{\mathcal{F}}\left(Q P^{\prime}\right)$ of order prime to $p$ with $\left.\alpha\right|_{P^{\prime}}=\operatorname{Id}_{P^{\prime}}$. Note that the set of $\mathcal{F}$-quasicentric subgroups of $S$ is closed under $\mathcal{F}$-conjugation and overgroups.

There is also a homotopy theoretic characterization of $\mathcal{F}$-quasicentric subgroups. If we define a map $f: X \rightarrow Y$ to be quasicentric if the homotopy fibre of the map $f_{\sharp}: \operatorname{Map}(X, X)_{\operatorname{Id}_{X}} \longrightarrow \operatorname{Map}(X, Y)_{f}$ is homotopically discrete, then it turns out that $P \leq S$ is $\mathcal{F}$-quasicentric in $(S, \mathcal{F}, \mathcal{L})$ if and only if the natural map $\left.f\right|_{B P}: B P \longrightarrow|\mathcal{L}|_{p}^{\wedge}$ is quasicentric.

Proposition 3.2. For any $p$-local finite group $(S, \mathcal{F}, \mathcal{L})$ and any $P \leq S$, the following are equivalent:

(a) $P$ is $\mathcal{F}$-quasicentric.

(b) There is a fully centralized subgroup $P^{\prime} \leq S$ which is $\mathcal{F}$-conjugate to $P$ and such that

$$
\operatorname{Map}\left(B P,|\mathcal{L}|_{p}^{\wedge}\right)_{\left.f\right|_{B P}} \simeq \operatorname{Map}\left(B P^{\prime},|\mathcal{L}|_{p}^{\wedge}\right)_{\left.f\right|_{B P^{\prime}}} \simeq B C_{S}\left(P^{\prime}\right) .
$$

(c) The homotopy fibre of the map $\operatorname{Map}(B P, B P)_{\operatorname{Id}_{B P}} \longrightarrow \operatorname{Map}\left(B P,|\mathcal{L}|_{p}^{\wedge}\right)_{\left.f\right|_{B P}}$ is homotopically discrete.

(d) $\operatorname{Map}\left(B P,|\mathcal{L}|_{p}^{\wedge}\right)_{\left.f\right|_{B P}}$ is an Eilenberg-MacLane space $K(G, 1)$.

Proof. $\quad((\mathrm{a}) \Rightarrow(\mathrm{b}))$ follows by definition of $\mathcal{F}$-quasicentric and [5 Theorem 6.3].

$((\mathrm{b}) \Rightarrow(\mathrm{c}))$ and $((\mathrm{c}) \Rightarrow(\mathrm{d}))$ follow from the long exact sequence of homotopy groups of the relevant fibration because $\operatorname{Map}(B P, B P)_{\operatorname{Id}_{B P}} \simeq B Z(P)$.

Finally we prove that $((\mathrm{d}) \Rightarrow(\mathrm{a}))$. Let $P^{\prime}$ be a fully centralized subgroup of $S$ which is $\mathcal{F}$-conjugate to $P$. By [ [5 Theorem 6.3], we have that

$$
\left|C_{\mathcal{L}}\left(P^{\prime}\right)\right|_{p}^{\wedge} \simeq \operatorname{Map}\left(B P^{\prime},|\mathcal{L}|_{p}^{\wedge}\right)_{\left.f\right|_{B P^{\prime}}} \simeq \operatorname{Map}\left(B P,|\mathcal{L}|_{p}^{\wedge}\right)_{\left.f\right|_{B P}} \simeq K(G, 1) .
$$

In particular, $G \cong \pi_{1}\left(\left|C_{\mathcal{L}}\left(P^{\prime}\right)\right|_{p}^{\wedge}\right)$ is a finite $p$-group, and then the fusion system $C_{\mathcal{F}}\left(P^{\prime}\right)$ coincides with the fusion system of $G$ (see [5, Theorem 7.4]).

We now turn to quasicentric linking systems; i.e., linking systems associated to a saturated fusion system $\mathcal{F}$ whose objects are the $\mathcal{F}$-quasicentric subgroups. These 
are defined in essentially the same way as centric linking systems; there is just one extra axiom which is needed.

Definition 3.3. Let $\mathcal{F}$ be a saturated fusion system over a p-group $S$. A quasicentric linking system associated to $\mathcal{F}$ consists of a category $\mathcal{L}^{q}$ whose objects are the $\mathcal{F}$-quasicentric subgroups of $S$, together with a functor $\pi: \mathcal{L}^{q} \longrightarrow \mathcal{F}^{q}$, and distinguished monomorphisms $P \cdot C_{S}(P) \stackrel{\delta_{P}}{\longrightarrow} \operatorname{Aut}_{\mathcal{L}^{q}}(P)$, which satisfy the following conditions.

$(\mathrm{A})_{q} \pi$ is the identity on objects and surjective on morphisms. For each pair of objects $P, Q$ in $\mathcal{L}^{q}$ such that $P$ is fully centralized in $\mathcal{F}, C_{S}(P)$ acts freely on $\operatorname{Mor}_{\mathcal{L}^{q}}(P, Q)$ by composition (upon identifying $C_{S}(P)$ with $\delta_{P}\left(C_{S}(P)\right) \leq$ $\left.\operatorname{Aut}_{\mathcal{L}^{q}}(P)\right)$, and $\pi$ induces a bijection

$$
\operatorname{Mor}_{\mathcal{L}^{q}}(P, Q) / C_{S}(P) \stackrel{\cong}{\longrightarrow} \operatorname{Hom}_{\mathcal{F}}(P, Q) .
$$

$(\mathrm{B})_{q}$ For each $\mathcal{F}$-quasicentric subgroup $P \leq S$ and each $g \in P, \pi$ sends $\delta_{P}(g) \in$ $\operatorname{Aut}_{\mathcal{L}^{q}}(P)$ to $c_{g} \in \operatorname{Aut}_{\mathcal{F}}(P)$.

$(\mathrm{C})_{q}$ For each $f \in \operatorname{Mor}_{\mathcal{L}^{q}}(P, Q)$ and each $g \in P$, the following square commutes in $\mathcal{L}^{q}$ :

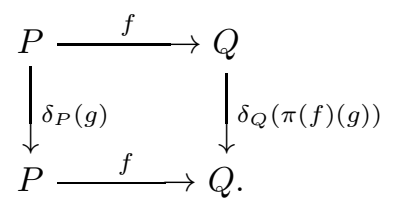

$(\mathrm{D})_{q}$ For each $\mathcal{F}$-quasicentric subgroup $P \leq S$, there is some $\iota_{P} \in \operatorname{Mor}_{\mathcal{L}^{q}}(P, S)$ such that $\pi\left(\iota_{P}\right)=\operatorname{incl}_{P}^{S} \in \operatorname{Hom}(P, S)$, and such that for each $g \in P \cdot C_{S}(P)$, $\delta_{S}(g) \circ \iota_{P}=\iota_{P} \circ \delta_{P}(g)$ in $\operatorname{Mor}_{\mathcal{L}^{q}}(P, S)$.

Note that point $(\mathrm{D})_{q}$ follows from $(\mathrm{C})_{q}$ if $P$ is $\mathcal{F}$-centric. This is why it does not appear in the definition of a centric linking system (Definition [1.4).

When $\mathcal{L}$ and $\mathcal{L}^{q}$ are centric and quasicentric linking systems associated to the same fusion system $\mathcal{F}$, we say that $\mathcal{L}^{q}$ extends $\mathcal{L}$ if $\mathcal{L}$ is isomorphic to a full subcategory of $\mathcal{L}^{q}$ in a way which is consistent with the projection functors and the distinguished monomorphisms.

We first show how to construct a quasicentric linking system which extends a given centric linking system. One way to do this is provided by [5 $\S 7]$. There, a (discrete) category $\mathcal{L}_{S, f}(X)$ is associated to any triple $(X, S, f)$, where $X$ is a space, $S$ is a $p$-group, and $f: B S \longrightarrow X$ is a map. We recall this construction in the case where $f$ is the natural inclusion of $B S$ into $X=|\mathcal{L}|_{p}^{\wedge}\left(f=\left|\theta_{S}\right|_{p}^{\wedge}\right.$ as defined in the next paragraph). As we will see, $\mathcal{L}_{S, f}\left(|\mathcal{L}|_{p}^{\wedge}\right)$ is then an extension of $\mathcal{L}$ containing all subgroups of $S$ as objects.

Let $(S, \mathcal{F}, \mathcal{L})$ be a $p$-local finite group, and let $\pi: \mathcal{L} \longrightarrow \mathcal{F}^{c}$ be the projection functor. For each subgroup $P \leq S$, let $\mathcal{B}(P)$ be the category with one object $o_{P}$ and with $\operatorname{End}_{\mathcal{B}(P)}\left(o_{P}\right)=P$, and identify $B P=|\mathcal{B}(P)|$. We let $\check{g}$ denote the morphism in $\mathcal{B}(P)$ corresponding to $g \in P$. Let

$$
\theta_{P}: \mathcal{B}(P) \longrightarrow \mathcal{L}
$$


be the functor which sends $o_{P}$ to $P$, and sends a morphism $\check{g}$ (for $g \in P$ ) to $\delta_{P}(g) \in \operatorname{Aut}_{\mathcal{L}}(P)$. This induces natural maps $\left|\theta_{P}\right|_{p}^{\wedge}: B P \longrightarrow|\mathcal{L}|_{p}^{\wedge}$. For each $\varphi \in$ $\operatorname{Hom}_{\mathcal{L}}(P, Q)$, we can view $\pi(\varphi) \in \operatorname{Hom}_{\mathcal{F}}(P, Q)$ as a functor $\mathcal{B}(P) \rightarrow \mathcal{B}(Q)$. Let

$$
\eta_{\varphi}: \theta_{P} \longrightarrow \theta_{Q} \circ \pi(\varphi)
$$

be the natural transformation of functors given by

$$
\theta_{P}\left(o_{P}\right)=P \stackrel{\varphi}{\longrightarrow} Q=\theta_{Q}\left(\pi(\varphi)\left(o_{P}\right)\right) .
$$

This defines an explicit homotopy $\left|\eta_{\varphi}\right|: B P \times I \longrightarrow|\mathcal{L}|_{p}^{\wedge}$ between $\left|\theta_{P}\right|_{p}^{\wedge}$ and $\left|\theta_{Q}\right|_{p}^{\wedge}$ 。 $B \varphi$. If for each $\mathcal{F}$-centric subgroup $P \leq S$, we choose a morphism $\iota_{P} \in \operatorname{Mor}_{\mathcal{L}}(P, S)$ which is sent to the inclusion of $P$ in $S$ by the projection functor to $\mathcal{F}$, we obtain a fixed collection of natural transformations $\eta_{\iota_{P}}$, and induced homotopies $\left|\eta_{\iota_{P}}\right|: B P \times$ $I \longrightarrow|\mathcal{L}|_{p}^{\wedge}$ from $\left|\theta_{P}\right|_{p}^{\wedge}$ to the restriction $\left.\left|\theta_{S}\right|_{p}^{\wedge}\right|_{B P}$

Write $f=\left|\theta_{S}\right|_{p}^{\wedge}$ for short. $\mathcal{L}_{S, f}\left(|\mathcal{L}|_{p}^{\wedge}\right)$ is defined as the category whose objects are the subgroups of $S$, and where morphisms are

$$
\begin{aligned}
& \operatorname{Mor}_{\mathcal{L}_{S, f}\left(|\mathcal{L}|_{p}\right)}(P, Q) \\
& =\left\{(\varphi,[H]) \mid \varphi \in \operatorname{Hom}(P, Q),[H] \in \operatorname{Mor}_{\pi\left(\operatorname{Map}\left(B P,|\mathcal{L}|_{p}^{\wedge}\right)\right)}\left(\left.f\right|_{B P},\left.f\right|_{B Q} \circ B \varphi\right)\right\} .
\end{aligned}
$$

Here, $\pi$ denotes the fundamental groupoid functor. A functor

$$
\xi_{\mathcal{L}}: \mathcal{L} \longrightarrow \mathcal{L}_{S, f}\left(|\mathcal{L}|_{p}^{\wedge}\right)
$$

is also defined as follows. On objects, $\xi_{\mathcal{L}}$ is the inclusion. For each $\varphi \in \operatorname{Mor}_{\mathcal{L}}(P, Q)$, $\xi_{\mathcal{L}}(\varphi)=\left(\pi_{P, Q}(\varphi),\left[H_{\varphi}\right]\right)$, where $H_{\varphi}$ is the homotopy $B P \times I \longrightarrow|\mathcal{L}|_{p}^{\wedge}$ defined by

$$
H_{\varphi}(x, t)= \begin{cases}\left|\eta_{\iota_{P}}\right|(x, 1-3 t) & 0 \leq t \leq \frac{1}{3} \\ \left|\eta_{\varphi}\right|(x, 3 t-1) & \frac{1}{3} \leq t \leq \frac{2}{3} \\ \left|\eta_{\iota_{Q}}\right|(B \varphi(x), 3 t-2) & \frac{2}{3} \leq t \leq 1 .\end{cases}
$$

By [5. Proposition 7.3], $\xi_{\mathcal{L}}$ defines an equivalence of categories to the full subcategory $\mathcal{L}_{S, f}^{c}\left(|\mathcal{L}|_{p}^{\wedge}\right) \subseteq \mathcal{L}_{S, f}\left(|\mathcal{L}|_{p}^{\wedge}\right)$ whose objects are the $\mathcal{F}$-centric subgroups of $|\mathcal{L}|_{p}^{\wedge}$. In this sense, we say that $\mathcal{L}_{S, f}\left(|\mathcal{L}|_{p}^{\wedge}\right)$ is an extension of $\mathcal{L}$.

Proposition 3.4. Fix a p-local finite group $(S, \mathcal{F}, \mathcal{L})$, and let $f: B S \longrightarrow|\mathcal{L}|_{p}^{\wedge}$ be as defined above. Let $\mathcal{L}^{q} \subseteq \mathcal{L}_{S, f}\left(|\mathcal{L}|_{p}^{\wedge}\right)$ be the full subcategory whose objects are the $\mathcal{F}$-quasicentric subgroups of $S$, and regard $\mathcal{L}$ as a full subcategory of $\mathcal{L}^{q}$ via $\xi_{\mathcal{L}}$. Let

$$
\pi: \mathcal{L}^{q} \longrightarrow \mathcal{F}^{q}
$$

be the functor which sends an $\mathcal{F}$-quasicentric subgroup to itself, and which sends a morphism $(\varphi,[H])$ to $\varphi$. For each object $P$ in $\mathcal{L}^{q}$, define the distinguished monomorphism

$$
\delta_{P}: P \cdot C_{S}(P) \longrightarrow \operatorname{Aut}_{\mathcal{L}^{q}}(P)
$$

by sending $g \in P \cdot C_{S}(P)$ to $\left(c_{g},\left[H_{g}\right]\right)$, where $c_{g}$ is conjugation by $g$ restricted to $P$ and $H_{g}$ is the homotopy $B P \times I \stackrel{\left|\eta_{g}\right|}{\longrightarrow} B S \stackrel{f}{\longrightarrow}|\mathcal{L}|_{p}^{\wedge}$ induced by the natural transformation $\mathrm{Id} \stackrel{\eta_{g}}{\longrightarrow} c_{g}$ which sends the unique object of $\mathcal{B}(P)$ to the morphism $\check{g}$ of $\mathcal{B}(S)$. Then these structures make $\mathcal{L}^{q}$ into a quasicentric linking system associated to $\mathcal{F}$ which extends $\mathcal{L}$. 
Proof. If $P \leq S$ is $\mathcal{F}$-quasicentric and fully centralized in $\mathcal{F}$, then

$$
\operatorname{Map}\left(B P,|\mathcal{L}|_{p}^{\wedge}\right)_{f \mid B P} \simeq\left|C_{\mathcal{L}}(P)\right|_{p}^{\wedge} \simeq B C_{S}(P):
$$

the first equivalence by [5. Theorem 6.3] and the second since $P$ is $\mathcal{F}$-quasicentric. Hence by definition of the category $\mathcal{L}_{S, f}\left(|\mathcal{L}|_{p}^{\wedge}\right), C_{S}(P)$ acts freely on $\operatorname{Mor}_{\mathcal{L}^{q}}(P, Q)$ (for any $Q$ ) with orbit set $\operatorname{Hom}_{\mathcal{F}}(P, Q)$, and this proves axiom $(\mathrm{A})_{q}$. This also shows that $\xi_{\mathcal{L}}$ embeds $\mathcal{L}$ as a full subcategory of $\mathcal{L}^{q}$.

Axiom $(\mathrm{B})_{q}$ follows immediately from the definitions. The proof of $(\mathrm{C})_{q}$ is identical to the argument used to prove $(\mathrm{C})$ in the proof of [5 Theorem 7.5]. Point (D) ${ }_{q}$ follows immediately from the construction, upon setting $\iota_{P}=\left(\operatorname{incl}_{P}^{S},[c]\right)$, where $c$ denotes the constant path with value $\left.f\right|_{B P} \in \operatorname{Map}\left(B P,|\mathcal{L}|_{p}^{\wedge}\right)$.

We are now ready to state the main result of this section:

TheOREm 3.5. Let $(S, \mathcal{F}, \mathcal{L})$ be a p-local finite group, and let $\mathcal{L}^{q}$ be a quasicentric linking system associated to $\mathcal{F}$ which extends $\mathcal{L}$. Let $\mathcal{L}^{\prime} \subseteq \mathcal{L}^{q}$ be any full subcategory which contains all $\mathcal{F}$-radical $\mathcal{F}$-centric subgroups of $S$. Then the inclusions of $\mathcal{L}^{\prime}$ and $\mathcal{L}$ in $\mathcal{L}^{q}$ induce homotopy equivalences $\left|\mathcal{L}^{\prime}\right| \simeq\left|\mathcal{L}^{q}\right| \simeq|\mathcal{L}|$.

Theorem 3.5 is an immediate consequence of Proposition 3.11 below. The rest of the section is directed towards the proof of that proposition. We first prove some lemmas that will provide us with a better understanding of morphism sets in $\mathcal{L}^{q}$.

Lemma 3.6. Fix a p-local finite group $(S, \mathcal{F}, \mathcal{L})$, let $\mathcal{L}^{q}$ be a quasicentric linking system associated to $\mathcal{F}$ which extends $\mathcal{L}$, and let $\pi: \mathcal{L}^{q} \longrightarrow \mathcal{F}^{q}$ be the projection. Fix $\mathcal{F}$-quasicentric subgroups $P, Q, R$ in $S$. Let $\varphi \in \operatorname{Mor}_{\mathcal{L}^{q}}(P, R)$ and $\psi \in \operatorname{Mor}_{\mathcal{L}^{q}}(Q, R)$ be any pair of morphisms such that $\operatorname{Im}(\pi(\varphi)) \leq \operatorname{Im}(\pi(\psi))$. Then there is a unique morphism $\chi \in \operatorname{Mor}_{\mathcal{L}^{q}}(P, Q)$ such that $\varphi=\psi \circ \chi$.

Proof. By definition of a fusion system (every morphism is the composite of an isomorphism followed by an inclusion), there is a unique morphism $\bar{\chi} \in \operatorname{Hom}_{\mathcal{F}}(P, Q)$ such that $\pi(\varphi)=\pi(\psi) \circ \bar{\chi}$. Let $\chi^{\prime} \in \operatorname{Mor}_{\mathcal{L}^{q}}(P, Q)$ be any morphism such that $\pi\left(\chi^{\prime}\right)=\bar{\chi}$. Choose a fully centralized group $P^{\prime}$ in the $\mathcal{F}$-conjugacy class of $P$ and a particular $\alpha \in \operatorname{Iso}_{\mathcal{L}^{q}}\left(P^{\prime}, P\right)$. Then by $(\mathrm{A})_{q}$, there is a unique element $g \in C_{S}\left(P^{\prime}\right)$ such that $\varphi \circ \alpha=\psi \circ \chi^{\prime} \circ \alpha \circ \delta_{P^{\prime}}(g)$, and we can define $\chi=\chi^{\prime} \circ \alpha \circ \delta_{P^{\prime}}(g) \circ \alpha^{-1}$.

If $\chi_{1} \in \operatorname{Mor}_{\mathcal{L}^{q}}(P, Q)$ is any other morphism such that $\varphi=\psi \circ \chi_{1}$, then $\pi(\chi)=$ $\pi\left(\chi_{1}\right)$, hence by $(\mathrm{A})_{q}$ again, there is a unique element $h \in C_{S}\left(P^{\prime}\right)$ such that $\chi \circ \alpha=$ $\chi_{1} \circ \alpha \circ \delta_{P^{\prime}}(h)$; and since $\psi \circ \chi_{1} \circ \alpha=\psi \circ \chi \circ \alpha=\psi \circ \chi_{1} \circ \alpha \circ \delta_{P^{\prime}}(h)$, and the action of $C_{S}\left(P^{\prime}\right)$ on $\operatorname{Mor}_{\mathcal{L}^{q}}\left(P^{\prime}, Q\right)$ is free, we obtain $h=1$ and then $\chi=\chi_{1}$.

Lemma 3.7. Fix a p-local finite group $(S, \mathcal{F}, \mathcal{L})$. Let $\mathcal{L}^{q}$ be quasicentric linking system associated to $\mathcal{F}$ which extends $\mathcal{L}$, and let $\pi: \mathcal{L}^{q} \longrightarrow \mathcal{F}^{q}$ be the projection. Fix a choice of an inclusion morphism $\iota_{P} \in \operatorname{Mor}_{\mathcal{L}^{q}}(P, S)$ for each $\mathcal{F}$-quasicentric subgroup $P \leq S$, such that $\pi\left(\iota_{P}\right)=\operatorname{incl} \in \operatorname{Hom}(P, S)$, such that the conclusion of $(D)_{q}$ holds if $P$ is not $\mathcal{F}$-centric, and where $\iota_{S}=\operatorname{Id}_{S}$. Then, there are unique 
injections

$$
\delta_{P, Q}: N_{S}(P, Q) \longrightarrow \operatorname{Mor}_{\mathcal{L}^{q}}(P, Q),
$$

for all $\mathcal{F}$-quasicentric subgroups $P, Q \leq S$, such that:

(a) $\pi\left(\delta_{P, Q}(g)\right)=c_{g} \in \operatorname{Hom}(P, Q)$, for all $g \in N_{S}(P, Q)$,

(b) $\delta_{P, S}(1)=\iota_{P}$ and $\delta_{P, P}(g)=\delta_{P}(g)$, for all $g \in P \cdot C_{S}(P)$,

(c) $\delta_{Q, R}(h) \circ \delta_{P, Q}(g)=\delta_{P, R}(h g)$, for all $g \in N_{S}(P, Q)$ and $h \in N_{S}(Q, R)$.

Proof. For each $P$ and $Q$, and each $g \in N_{S}(P, Q)$, there is by Lemma 3.6 a unique morphism $\delta_{P, Q}(g)$ such that

$$
\delta_{S}(g) \circ \iota_{P}=\iota_{Q} \circ \delta_{P, Q}(g) .
$$

We take this as the definition of the maps $\delta_{P, Q}$. Property (a) follows from $(\mathrm{B})_{q}$, (b) follows by definition and the assumptions about $\iota_{P}$, and (c) follows from Lemma [3.6. (Compare with [5, Proposition 1.11] and its proof.)

For the rest of the section, whenever we are given a $p$-local finite group $(S, \mathcal{F}, \mathcal{L})$, we assume that we have chosen morphisms $\iota_{P} \in \operatorname{Mor}_{\mathcal{L}^{q}}(P, S)$, for each object $P$, such that $\pi\left(\iota_{P}\right)$ is the inclusion and the conclusion of $(\mathrm{D})_{q}$ holds. Then for each $P \leq Q$ in $\mathcal{L}^{q}$, we let $\iota_{P}^{Q} \in \operatorname{Mor}_{\mathcal{L}^{q}}(P, Q)$ be the unique morphism such that $\iota_{P}=$ $\iota_{Q} \circ \iota_{P}^{Q}$ (Lemma 3.6). If $\varphi \in \operatorname{Mor}_{\mathcal{L}^{q}}(P, Q)$, and $P^{\prime} \leq P$ and $Q^{\prime} \leq Q$ are quasicentric subgroups such that $\pi(\varphi)\left(P^{\prime}\right) \leq Q^{\prime}$, then we write $\left.\varphi\right|_{P^{\prime}} ^{Q^{\prime}} \in \operatorname{Mor}_{\mathcal{L}^{q}}\left(P^{\prime}, Q^{\prime}\right)$ for the "restriction" of $\varphi$ : the unique morphism such that $\left.\iota_{Q^{\prime}}^{Q} \circ \varphi\right|_{P^{\prime}} ^{Q^{\prime}}=\varphi \circ \iota_{P^{\prime}}^{P}$ (Lemma 3.6 again). We also write $\left.\varphi\right|_{P^{\prime}}=\left.\varphi\right|_{P^{\prime}} ^{Q^{\prime}}$ when the target group $Q^{\prime}$ is clear from the context.

LEMMA 3.8. Fix a saturated fusion system $\mathcal{F}$ over a p-group $S$, and let $Q \leq S$ be an $\mathcal{F}$-quasicentric subgroup. Let $P \leq S$ be such that $Q \triangleleft P$, and let $\varphi, \varphi^{\prime} \in$ $\operatorname{Hom}_{\mathcal{F}}(P, S)$ be such that $\left.\varphi\right|_{Q}=\left.\varphi^{\prime}\right|_{Q}$, and $\varphi(Q)=\varphi^{\prime}(Q)$ is fully centralized in $\mathcal{F}$. Then there is $x \in C_{S}(\varphi(Q))$ such that $\varphi^{\prime}=c_{x} \circ \varphi$.

Proof. Upon replacing $P$ by $\varphi^{\prime}(P)$ and $Q$ by $\varphi(Q)=\varphi^{\prime}(Q)$, we can assume that $\varphi^{\prime}=\operatorname{incl}_{P}^{S}$ and $\left.\varphi\right|_{Q}=\operatorname{Id}_{Q}$. We are thus reduced to the case where $Q$ is fully centralized and $\varphi^{\prime}$ is the inclusion of $P$ in $S$.

The idea of the proof is to show that for some $x \in C_{S}(Q)$, we can extend $\varphi \circ c_{x}$ to some $\bar{\varphi} \in \operatorname{Hom}_{\mathcal{F}}(\bar{P}, S)$, for some $\bar{P} \geq P$, such that $\left.\bar{\varphi}\right|_{\bar{Q}}=\operatorname{Id}_{\bar{Q}}$ where $Q \supsetneqq \bar{Q} \triangleleft \bar{P}$. The lemma then follows by downward induction on $|Q|$. Recall that the lemma holds when $Q$ is $\mathcal{F}$-centric by [5, Proposition A.8].

By definition of an $\mathcal{F}$-quasicentric subgroup, $\left.\varphi\right|_{C_{P}(Q)}$ is conjugation by some element $x \in C_{S}(Q)$. So after composing with $c_{x}$, we can assume that $\left.\varphi\right|_{C_{P}(Q) \cdot Q}=\operatorname{Id}$. We are thus done if $C_{P}(Q) \cdot Q \supsetneqq Q$ by taking $\bar{P}=P$ and $\bar{Q}=C_{P}(Q) \cdot Q$.

Assume now that $C_{P}(Q) \leq Q$. Set $K=\operatorname{Aut}_{P}(Q)$. As in [5 Appendix A], we write

$$
N_{S}^{K}(Q)=\left\{x \in N_{S}(Q) \mid c_{x} \in K\right\}
$$

and let $N_{\mathcal{F}}^{K}(Q)$ be the fusion system over $N_{S}^{K}(Q)$ whose morphisms are defined (for 
$\left.P, P^{\prime} \leq N_{S}^{K}(Q)\right)$ by

$$
\begin{aligned}
& \operatorname{Hom}_{N_{\mathcal{F}}^{K}(Q)}\left(P, P^{\prime}\right) \\
& \quad=\left\{\varphi \in \operatorname{Hom}_{\mathcal{F}}\left(P, P^{\prime}\right)|\psi|_{P}=\varphi,\left.\psi\right|_{Q} \in K \text {, some } \psi \in \operatorname{Hom}_{\mathcal{F}}\left(P Q, P^{\prime} Q\right)\right\} .
\end{aligned}
$$

Then $P, \varphi(P)$, and $C_{S}(Q)$ are all contained in $N_{S}^{K}(Q)$. If $Q$ is not fully $K$ normalized in $\mathcal{F}$, then there is some $\psi \in \operatorname{Hom}_{\mathcal{F}}\left(N_{S}^{K}(Q), S\right)$ such that $\psi(Q)$ is fully $\psi K \psi^{-1}$-normalized in $\mathcal{F}$ (see [5. Proposition A.2(b)]); and upon replacing all of these subgroups by their images under $\psi$, we are reduced to the case where $Q$ is fully $K$-normalized in $\mathcal{F}$. The fusion system $N_{\mathcal{F}}^{K}(Q)$ is saturated by $\mathbf{5}$ Proposition A.6]; and upon replacing $\mathcal{F}$ by $N_{\mathcal{F}}^{K}(Q)$ we can assume that $S=N_{S}^{K}(Q)=P \cdot C_{S}(Q)$ and $\mathcal{F}=N_{\mathcal{F}}^{K}(Q)$. In particular, each $\alpha \in \operatorname{Hom}_{\mathcal{F}}\left(R, R^{\prime}\right)$ extends to a morphism in $\operatorname{Hom}_{\mathcal{F}}\left(R Q, R^{\prime} Q\right)$ whose restriction to $Q$ is conjugation by some element of $P$.

Fix $\psi \in \operatorname{Hom}_{\mathcal{F}}(P, S)$ such that $\psi(P)$ is fully normalized in $\mathcal{F}$. Since $\left.\psi\right|_{Q}$ is conjugation by an element $g \in P$, we can replace $\psi$ by $\psi \circ c_{g}^{-1}$, and thus arrange that $\left.\psi\right|_{Q}=$ Id. If $\psi$ and $\psi \circ \varphi^{-1}$ are both conjugation by some element of $C_{S}(Q)$, then so is $\varphi$; so it suffices to prove the result under the assumption that $\varphi(P)$ is fully normalized in $\mathcal{F}$.

Now, $\left(C_{S}(Q) \cdot Q\right) / Q$ is a nontrivial normal subgroup of $N_{S}(Q) / Q=S / Q$. So there is an element $x \in C_{S}(Q) \backslash Q$ such that $1 \neq x Q \in Z(S / Q)$. Then $x \in N_{S}(P)$, and acts via the identity on $Q$ and on $P / Q$. Thus

$$
c_{x} \in \operatorname{Ker}\left[\operatorname{Aut}_{\mathcal{F}}(P) \longrightarrow \operatorname{Aut}_{\mathcal{F}}(Q) \times \operatorname{Aut}(P / Q)\right],
$$

a normal $p$-subgroup of $\operatorname{Aut}_{\mathcal{F}}(P)$ (see 10 Corollary 5.3.3]). Also, $\operatorname{Aut}_{S}(\varphi(P)) \in$ $\operatorname{Syl}_{p}\left(\operatorname{Aut}_{\mathcal{F}}(\varphi(P))\right)$ since $\varphi(P)$ is fully normalized. Hence $\varphi c_{x} \varphi^{-1} \in \operatorname{Aut}_{S}(\varphi(P))$ (after replacing $\varphi$ by $\varphi \circ \xi$ where $\xi \in \operatorname{Aut}_{\mathcal{F}}(\varphi(P))$ if necessary). Thus, $x \in N_{\varphi}$ and $Q \supsetneqq N_{\varphi}$. By (II), $\varphi$ extends to $\bar{\varphi} \in \operatorname{Hom}_{\mathcal{F}}\left(N_{\varphi}, S\right)$. Now set $\bar{P}=N_{\varphi} \cap N_{S}(Q)=N_{\varphi}$ and $\bar{Q}=C_{\bar{P}}(Q) \cdot Q$.

By construction, $x \in \bar{Q} \backslash Q$. Since $Q$ is $\mathcal{F}$-quasicentric, $\left.\bar{\varphi}\right|_{C_{\bar{P}}(Q)}$ is conjugation by some element $g \in C_{S}(Q)$. So we can replace $\bar{\varphi}$ by $\bar{\varphi} \circ\left(c_{g}\right)^{-1}$, and thus arrange that $\left.\bar{\varphi}\right|_{\bar{Q}}=\operatorname{Id}_{\bar{Q}}$. Since $\bar{Q} \supsetneqq Q$ and $\bar{Q} \triangleleft \bar{P}$, this finishes the induction step.

The next lemma can be thought of as a "lifting" of the last one to quasicentric linking systems. It says that all inclusions in $\mathcal{L}^{q}$ are epimorphisms in the categorical sense.

Lemma 3.9. Fix a p-local finite group $(S, \mathcal{F}, \mathcal{L})$, and let $\mathcal{L}^{q}$ be a quasicentric linking system associated to $\mathcal{F}$ which extends $\mathcal{L}$. Assume $Q \leq P \leq S$ and $R \leq S$ are $\mathcal{F}$-quasicentric, and let $\varphi, \varphi^{\prime} \in \operatorname{Mor}_{\mathcal{L}^{q}}(P, R)$ be two morphisms such that $\varphi \circ \iota_{Q}^{P}=$ $\varphi^{\prime} \circ \iota_{Q}^{P}$. Then $\varphi=\varphi^{\prime}$.

Proof. Since there is always a subnormal series $Q=Q_{0} \triangleleft Q_{1} \triangleleft \cdots \triangleleft Q_{k}=P$, it suffices to prove the lemma when $Q$ is normal in $P$. So we assume this from now on.

It will be convenient, throughout the proof, to write $\widehat{\alpha}=\pi(\alpha) \in \operatorname{Mor}(\mathcal{F})$ for any $\alpha \in \operatorname{Mor}\left(\mathcal{L}^{q}\right)$. By Lemma 3.6] $\varphi=\varphi^{\prime}$ if and only if $\iota_{R}^{S} \circ \varphi=\iota_{R}^{S} \circ \varphi^{\prime} \in \operatorname{Mor}_{\mathcal{L}^{q}}(P, S)$, and similarly replacing $\varphi\left(\right.$ resp. $\left.\varphi^{\prime}\right)$ by $\varphi \circ \iota_{Q}^{P}\left(\right.$ resp. $\left.\varphi^{\prime} \circ \iota_{Q}^{P}\right)$. We can thus replace $R$ 
by any other subgroup of $S$ which contains the images of $\widehat{\varphi}$ and $\widehat{\varphi}^{\prime}$, and in particular assume that $R \leq N_{S}(\widehat{\varphi}(Q))$.

The proof itself will be divided in two steps: the first dealing with a restricted case, and the second reducing the general case to that in Step 1.

Step 1: Assume first that $Q=\widehat{\varphi}(Q)$ and is fully normalized, and that $P$ is fully centralized. Set $\varphi_{0}=\varphi \circ \iota_{Q}^{P}=\varphi^{\prime} \circ \iota_{Q}^{P}$. By condition (II) in Definition 1.3 (and since $Q=\widehat{\varphi}_{0}(Q)$ is fully centralized), there is $\psi \in \operatorname{Hom}_{\mathcal{L}}\left(P \cdot C_{S}(Q), S\right)$ such that $\left.\widehat{\psi}\right|_{Q}=\widehat{\varphi}_{0}$. Set $\varphi^{\prime \prime}=\left.\psi\right|_{P} \in \operatorname{Mor}_{\mathcal{L}^{q}}(P, S)$. Thus $\left.\widehat{\varphi}^{\prime \prime}\right|_{Q}=\widehat{\varphi}_{0}$, so there is a unique element $a \in C_{S}(Q)$ such that

$$
\left.\psi\right|_{Q}=\left.\varphi^{\prime \prime}\right|_{Q}=\iota_{R}^{S} \circ \varphi_{0} \circ \delta(a) .
$$

We will show that $\varphi=\varphi^{\prime}$ by comparing both to $\psi$ and $\varphi^{\prime \prime}$; the advantage of this is that condition (C) can be applied more easily to $\psi$.

By Lemma 3.8 there is some $x \in C_{S}(Q)(\widehat{\varphi}(Q)=Q)$ such that $c_{x} \circ \widehat{\varphi}=\widehat{\varphi}^{\prime \prime}$. Since $P$ is fully centralized, by condition $(\mathrm{A})_{q}$ in Definition 3.3 there is some $y \in C_{S}(P)$ such that

$\delta_{R, S}(x) \circ \varphi=\iota_{R}^{S} \circ \varphi^{\prime \prime} \circ \delta_{P}(y)=\left.\psi \circ \delta_{P \cdot C_{S}(Q)}(y)\right|_{P}=\left.\delta_{S}(\widehat{\psi}(y)) \circ \psi\right|_{P}=\delta_{S}(\widehat{\psi}(y)) \circ \varphi^{\prime \prime}$.

It follows that $\varphi^{\prime \prime}=\delta_{R, S}(z) \circ \varphi$, where $z=\widehat{\psi}(y)^{-1} \cdot x \in C_{S}(Q)$. Hence

$$
\begin{aligned}
& \delta_{R, S}\left(\widehat{\psi}(a)^{-1} \cdot z\right) \circ \varphi_{0}=\left.\delta_{S}(\widehat{\psi}(a))^{-1} \circ \varphi^{\prime \prime}\right|_{Q}=\left.\delta_{S}(\widehat{\psi}(a))^{-1} \circ \psi\right|_{Q} \\
& \quad=\left.\psi \circ \delta_{P \cdot C_{S}(Q)}(a)^{-1}\right|_{Q}=\left.\psi\right|_{Q} \circ \delta_{Q}(a)^{-1}=\varphi^{\prime \prime} \circ \iota_{Q}^{P} \circ \delta_{Q}(a)^{-1}=\iota_{R}^{S} \circ \varphi_{0} .
\end{aligned}
$$

Since $\varphi_{0}=\iota_{Q}^{R} \circ \omega$ for some $\omega \in \operatorname{Aut}_{\mathcal{L}^{q}}(Q)$, upon composing with $\omega^{-1}$, this shows that $\delta_{Q, S}\left(\widehat{\psi}(a)^{-1} \cdot z\right)=\iota_{Q}^{S}$, and hence that $z=\widehat{\psi}(a)$.

After making a similar argument involving $\varphi^{\prime}$, we now have

$$
\delta(\widehat{\psi}(a)) \circ \varphi=\varphi^{\prime \prime}=\delta(\widehat{\psi}(a)) \circ \varphi^{\prime},
$$

and this shows that $\varphi=\varphi^{\prime}$.

Step 2: (General case.) We first reduce the problem to the case in which $P$ is fully centralized. We choose an isomorphism $\xi \in \operatorname{Mor}_{\mathcal{L}^{q}}\left(P, P^{\prime}\right)$ such that $\widehat{\xi}(P)=P^{\prime}$ is fully centralized. Upon replacing $P$ by $P^{\prime}, \varphi$ by $\varphi \circ \xi^{-1}$, and $\varphi^{\prime}$ by $\varphi^{\prime} \circ \xi^{-1}$ we are now reduced to the case where $P$ is fully centralized in $\mathcal{F}$.

Set $Q^{\prime}=\widehat{\varphi}(Q)=\widehat{\varphi}^{\prime}(Q)$ for short; we now reduce the problem to the case in which $Q=Q^{\prime}$ and is fully normalized. Let $Q^{\prime \prime}$ be any fully normalized subgroup in the $\mathcal{F}$-conjugacy class of $Q$ (and of $Q^{\prime}$ ). By Lemma 2.3 (condition (IIB) holds), there are morphisms

$$
\beta \in \operatorname{Mor}_{\mathcal{L}^{q}}\left(N_{S}(Q), N_{S}\left(Q^{\prime \prime}\right)\right) \quad \text { and } \quad \beta^{\prime} \in \operatorname{Mor}_{\mathcal{L}^{q}}\left(N_{S}\left(Q^{\prime}\right), N_{S}\left(Q^{\prime \prime}\right)\right)
$$

such that $\widehat{\beta}(Q)=\widehat{\beta}^{\prime}\left(Q^{\prime}\right)=Q^{\prime \prime}$. Set $P^{\prime \prime}=\widehat{\beta}(P)$, and let $\beta_{0} \in \operatorname{Iso}_{\mathcal{L}^{q}}\left(P, P^{\prime \prime}\right)$ be the restriction of $\beta$ (i.e., by Lemma 3.6 the unique morphism such that $\iota_{P^{\prime \prime}}^{N_{S}\left(Q^{\prime \prime}\right)} \circ \beta_{0}=$ $\left.\beta \circ \iota_{P}^{N_{S}(Q)}\right)$. Set

$$
\psi=\beta^{\prime} \circ \iota_{R}^{N_{S}\left(Q^{\prime \prime}\right)} \circ \varphi \circ \beta_{0}^{-1}, \psi^{\prime}=\beta^{\prime} \circ \iota_{R}^{N_{S}\left(Q^{\prime \prime}\right)} \circ \varphi^{\prime} \circ \beta_{0}^{-1} \in \operatorname{Mor}_{\mathcal{L}^{q}}\left(P^{\prime \prime}, N_{S}\left(Q^{\prime \prime}\right)\right) .
$$

Then $\psi=\psi^{\prime}$ if and only if $\varphi=\varphi^{\prime}$, and $\psi \circ \iota_{Q^{\prime \prime}}^{P^{\prime \prime}}=\psi^{\prime} \circ \iota_{Q^{\prime \prime}}^{P^{\prime \prime}}$ if and only if $\varphi \circ \iota_{Q}^{P}=\varphi^{\prime} \circ \iota_{Q}^{P}$. Note that $P^{\prime \prime}$ is $\mathcal{F}$-conjugate to $P$ and the following inequality holds:

$$
\left|C_{S}(P)\right|=\left|C_{N_{S}(Q)}(P)\right| \leq\left|C_{N_{S}\left(Q^{\prime \prime}\right)}\left(P^{\prime \prime}\right)\right|=\left|C_{S}\left(P^{\prime \prime}\right)\right| .
$$


Since $P$ is fully centralized, it follows that $\left|C_{S}(P)\right|=\left|C_{S}\left(P^{\prime \prime}\right)\right|$ and $P^{\prime \prime}$ is also fully centralized.

Thus, upon replacing $(Q, P, R)$ by $\left(Q^{\prime \prime}, P^{\prime \prime}, N_{S}\left(Q^{\prime \prime}\right)\right), \varphi$ by $\psi$, and $\varphi^{\prime}$ by $\psi^{\prime}$, we are reduced to the case where $Q=\varphi(Q)$ is fully normalized and $P$ is fully centralized.

An immediate consequence of Lemmas 3.6 and 3.9 is:

Corollary 3.10. Let $\mathcal{L}^{q}$ be a quasicentric linking system associated to a saturated fusion system $\mathcal{F}$ over a p-group $S$. Then all morphisms in $\mathcal{L}^{q}$ are monomorphisms and epimorphisms in the categorical sense.

Proof. By the uniqueness in Lemma 3.6] $\psi \circ \chi=\psi \circ \chi^{\prime}$ in $\mathcal{L}^{q}$ implies $\chi=\chi^{\prime}$. Hence all morphisms in $\mathcal{L}^{q}$ are monomorphisms.

Since each morphism in $\mathcal{L}^{q}$ is the composite of an isomorphism followed by an inclusion, it suffices to prove that inclusions $\iota_{Q}^{P}$ are epimorphisms, and it clearly suffices to do this when $Q \triangleleft P$. So assume $P^{\prime} \leq S$ and $\varphi, \varphi^{\prime} \in \operatorname{Mor}_{\mathcal{L}^{q}}(P, R)$ are such that $\varphi \circ \iota_{Q}^{P}=\varphi^{\prime} \circ \iota_{Q}^{P}$. Then $\iota_{P^{\prime}}^{S} \circ \varphi=\iota_{P^{\prime}}^{S} \circ \varphi^{\prime}$ by Lemma 3.9 and so $\varphi=\varphi^{\prime}$ by Lemma 3.6

We are now ready to prove the following proposition, of which Theorem 3.5 is an immediate consequence.

Proposition 3.11. Let $(S, \mathcal{F}, \mathcal{L})$ be a p-local finite group, and let $\mathcal{L}^{q}$ be a quasicentric linking system associated to $\mathcal{F}$ which extends $\mathcal{L}$. Let $\mathcal{L}_{0} \subseteq \mathcal{L}^{q}$ be any full subcategory such that $\mathrm{Ob}\left(\mathcal{L}_{0}\right)$ is closed under $\mathcal{F}$-conjugacy. Let $P \in \operatorname{Ob}\left(\mathcal{L}^{q}\right)$ be maximal among those $\mathcal{F}$-quasicentric subgroups not in $\mathcal{L}_{0}$, and let $\mathcal{L}_{1} \subseteq \mathcal{L}^{q}$ be the full subcategory whose objects are the objects in $\mathcal{L}_{0}$ together with all subgroups $\mathcal{F}$ conjugate to $P$. Assume furthermore that $P$ is not $\mathcal{F}$-centric or not $\mathcal{F}$-radical. Then the inclusion of nerves $\left|\mathcal{L}_{0}\right| \subseteq\left|\mathcal{L}_{1}\right|$ is a homotopy equivalence.

Proof. Throughout the following proof, when working in any linking system, we assume that inclusion morphisms $\iota_{P}^{Q}$ have been chosen as in Lemma 3.7 By "extensions" and "restrictions" of morphisms we mean with respect to these inclusions. Also, for $\varphi \in \operatorname{Mor}_{\mathcal{L}^{q}}\left(Q, Q^{\prime}\right)$, we write $\operatorname{Im}(\varphi)=\operatorname{Im}(\pi(\varphi)) \leq Q^{\prime}$ and $\varphi(R)=\pi(\varphi)(R) \leq Q^{\prime}$ if $R \leq Q$.

We must show that the inclusion functor $\iota: \mathcal{L}_{0} \rightarrow \mathcal{L}_{1}$ induces a homotopy equivalence $\left|\mathcal{L}_{0}\right| \simeq\left|\mathcal{L}_{1}\right|$. By Quillen's Theorem A (see [20]), it will be enough to prove that the undercategory $Q \downarrow \iota$ is contractible (i.e., $|Q \downarrow \iota| \simeq *$ ) for each $Q$ in $\mathcal{L}_{1}$. This is clear when $Q$ is not isomorphic to $P$ (since $Q \downarrow \iota$ has initial object $(Q$, Id) in that case), so it suffices to consider the case $Q=P$. Since $P$ was arbitrarily chosen in its isomorphism class, we can also assume that $P$ is fully normalized.

Let

$$
\iota_{N}: \mathcal{L}_{0} \cap N_{\mathcal{L}^{q}}(P) \longrightarrow \mathcal{L}_{1} \cap N_{\mathcal{L}^{q}}(P)
$$

be the restriction of $\iota$. Consider the functor $i: P \downarrow \iota_{N} \rightarrow P \downarrow \iota$ induced by the inclu- 
sions $\mathcal{L}_{i} \cap N_{\mathcal{L}^{q}}(P) \rightarrow \mathcal{L}_{i}$ for $i=0,1$. We will first show that $|P \downarrow \iota| \simeq\left|P \downarrow \iota_{N}\right|$ and then that $\left|P \downarrow \iota_{N}\right| \simeq *$.

To prove the first statement, we construct a retraction functor $r: P \downarrow \iota \rightarrow P \downarrow \iota_{N}$ such that $r \circ i=\operatorname{Id}_{P \downarrow \iota_{N}}$, together with a natural transformation $\left(i \circ r \stackrel{\eta}{\longrightarrow} \operatorname{Id}_{P \downarrow i}\right)$. By Lemma 2.3 (condition (IIB)), for each $P^{\prime} \leq S$ which is $\mathcal{F}$-conjugate to $P$, there is a morphism in $\mathcal{F}$ from $N_{S}\left(P^{\prime}\right)$ to $N_{S}(P)$ which sends $P^{\prime}$ isomorphically to $P$. Hence upon lifting this to the linking system, we can choose a morphism

$$
\Phi_{P^{\prime}} \in \operatorname{Mor}_{\mathcal{L}^{q}}\left(N_{S}\left(P^{\prime}\right), N_{S}(P)\right)
$$

for each such $P^{\prime}$ which restricts to an isomorphism from $P^{\prime}$ to $P$. In particular, we set $\Phi_{P}=\operatorname{Id}_{N_{S}(P)}$.

For each nonisomorphism $\varphi \in \operatorname{Mor}_{\mathcal{L}^{q}}(P, Q)$, set $\widehat{r}(\varphi)=\Phi_{\varphi(P)}\left(N_{Q}(\varphi(P))\right) \supsetneqq P$. We can factor $\varphi$ as $\varphi=\eta(\varphi) \circ r(\varphi)$, where

$$
r(\varphi)=\iota_{P}^{\widehat{r}(\varphi)} \circ\left(\left.\Phi_{\varphi(P)}\right|_{\varphi(P)} \circ \varphi\right) \in \operatorname{Mor}_{N_{\mathcal{L}^{q}(P)}}(P, \widehat{r}(\varphi))
$$

and

$$
\eta(\varphi)=\iota_{\bar{Q}}^{Q} \circ\left(\Phi_{\varphi(P)} \mid \bar{Q}\right)^{-1} \in \operatorname{Mor}_{\mathcal{L}_{0}}(\widehat{r}(\varphi), Q),
$$

where $\bar{Q}=N_{Q}(\varphi(P))$. We define the functor $r: P \downarrow \iota \rightarrow P \downarrow \iota_{N}$ on objects by setting

$$
r(P \stackrel{\varphi}{\longrightarrow} Q)=(P \stackrel{r(\varphi)}{\longrightarrow} \widehat{r}(\varphi)) .
$$

For any morphism $\beta \in \operatorname{Mor}_{P \downarrow \mathcal{L}_{0}}\left((Q, \varphi),\left(Q^{\prime}, \varphi^{\prime}\right)\right)$; i.e., for any commutative square of the form

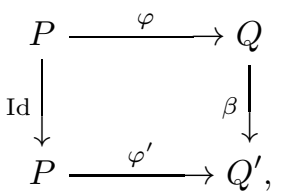

we claim there is a unique morphism $\widehat{r}(\beta)$ such that the two squares in the following diagram commute:

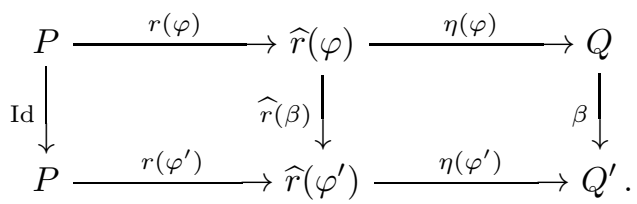

To see this, note that by commutativity of the square (1), $\beta$ sends $N_{Q}(\varphi(P))$ into $N_{Q^{\prime}}\left(\varphi^{\prime}(P)\right)$. Hence upon defining

$$
\widehat{r}(\beta) \stackrel{\text { def }}{=} \Phi_{\varphi^{\prime}(P)} \circ \beta \circ \Phi_{\varphi(P)}{ }^{-1},
$$

where the three morphisms are replaced by appropriate restrictions, we get $\widehat{r}(\beta)$ such that the right square in (2) commutes. Since the combination of the two squares commutes by assumption, we obtain that $\eta\left(\varphi^{\prime}\right) \circ \widehat{r}(\beta) \circ r(\varphi)=\eta\left(\varphi^{\prime}\right) \circ r\left(\varphi^{\prime}\right)$, and therefore $\widehat{r}(\beta) \circ r(\varphi)=r\left(\varphi^{\prime}\right)$ by Lemma 3.6. By the uniqueness of $\widehat{r}(\beta)$, it follows that this construction defines a functor, as well as a natural transformation $i \circ r \stackrel{\eta}{\longrightarrow} \operatorname{Id}_{P \downarrow i}$. Since $r \circ i=\operatorname{Id}_{P \downarrow i_{N}}$, this finishes the proof that $|P \downarrow \iota| \simeq\left|P \downarrow \iota_{N}\right|$.

It remains to prove that $|P \downarrow| \simeq *$. Set

$$
\widehat{P}=\left\{x \in N_{S}(P) \mid c_{x} \in O_{p}\left(\operatorname{Aut}_{\mathcal{F}}(P)\right)\right\} .
$$


Note that $\widehat{P} \geq P \cdot C_{S}(P)$, and hence $\widehat{P} \supsetneqq P$ if $P$ is not centric. Moreover, $\widehat{P} \supsetneqq P$ if $P$ is not radical, and thus $\widehat{P} \in \mathcal{L}_{0}$ in both cases covered by the hypotheses of the proposition. Since $P$ is normal in $\widehat{P}$, this last is an object in $\mathcal{L}_{0} \cap N_{\mathcal{L}^{q}}(P)$.

Recall that $\iota_{N}: \mathcal{L}_{0} \cap N_{\mathcal{L}^{q}}(P) \rightarrow \mathcal{L}_{1} \cap N_{\mathcal{L}^{q}}(P)$ denotes the inclusion. Let $i$ be the functor $i: \widehat{P} \downarrow \iota_{N} \rightarrow P \downarrow \iota_{N}$ which is induced by precomposing with the inclusion $\iota_{P}^{\widehat{P}} \in$ $\operatorname{Mor}_{\mathcal{L}^{q}}(P, \widehat{P})$. We show that $i$ induces a homotopy equivalence $|P \downarrow| \simeq\left|\widehat{P} \downarrow \iota_{N}\right|$, by defining a functor $r: P \downarrow \rightarrow \widehat{P} \downarrow \iota_{N}$ such that $r \circ i=\operatorname{Id}_{\widehat{P} \downarrow_{\iota}}$, and such that $i \circ r \simeq \operatorname{Id}_{P \downarrow \iota_{N}}$ (such that there is a natural transformation of functors from the identity to $i \circ r)$. Then $\left|P \downarrow \iota_{N}\right| \simeq|\widehat{P} \iota|$, and the last space is contractible since $\widehat{P} \in \mathcal{L}_{0} \cap N_{\mathcal{L}^{q}}(P)$. This will finish the proof.

Fix subgroups $Q, Q^{\prime} \leq N_{S}(P)$ containing $P$, and let $\varphi \in \operatorname{Mor}_{N_{\mathcal{L}}(P)}\left(Q, Q^{\prime}\right)$ be a morphism. Set $\alpha=\left.\pi(\varphi)\right|_{P} \in \operatorname{Aut}_{\mathcal{F}}(P)$ for short. Since $P$ is fully normalized, $\operatorname{Aut}_{S}(P) \in \operatorname{Syl}_{p}\left(\operatorname{Aut}_{\mathcal{F}}(P)\right)$, and hence $O_{p}\left(\operatorname{Aut}_{\mathcal{F}}(P)\right) \leq \operatorname{Aut}_{S}(P)$. It follows that

$$
N_{\alpha} \stackrel{\text { def }}{=}\left\{x \in N_{S}(P) \mid \alpha c_{x} \alpha^{-1} \in \operatorname{Aut}_{S}(P)\right\} \geq \widehat{P}
$$

and $N_{\alpha} \geq Q$ since $\alpha$ extends to $\pi(\varphi) \in \operatorname{Hom}_{\mathcal{F}}\left(Q, Q^{\prime}\right)$. Thus, since $P$ is fully centralized, $\alpha$ extends to some $\varphi^{\prime} \in \operatorname{Hom}_{\mathcal{F}}\left(Q \widehat{P}, Q^{\prime} \widehat{P}\right)$ by condition (II) in Definition 1.3 After possibly composing this extension with $\delta_{Q \widehat{P}}(x)$ for some element $x \in$ $C_{S}(P) \leq Q \widehat{P}$, we get a lifting $\widehat{\varphi} \in \operatorname{Mor}_{\mathcal{L}^{q}}\left(Q \widehat{P}, Q^{\prime} \widehat{P}\right)$ such that the following diagram commutes in $\mathcal{L}^{q}$ :

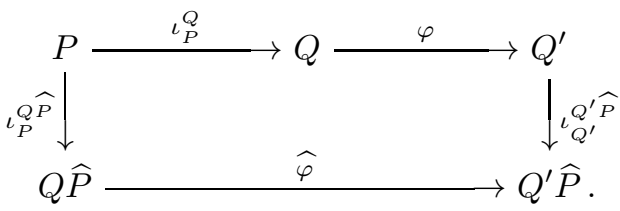

Hence by Lemma 3.9 $\widehat{\varphi} \circ \iota_{Q}^{Q P} \widehat{P}=\iota_{Q^{\prime}}^{Q^{\prime} \widehat{P}} \circ \varphi$. This lifting is unique by Corollary 3.10 and it lies in $\mathcal{L}_{0} \cap N_{\mathcal{L}^{q}}(P)$, or in $\mathcal{L}_{1} \cap N_{\mathcal{L}^{q}}(P)$ if $Q=P$.

The functor $r$ is defined on objects by setting

$$
r(P \stackrel{\varphi}{\longrightarrow} Q)=(\widehat{P} \stackrel{\widehat{\varphi}}{\longrightarrow} Q \widehat{P}) .
$$

If $\beta: Q \rightarrow Q^{\prime}$ is a morphism such that $\beta \circ \varphi=\varphi^{\prime}$, then we define $r(\beta)=\widehat{\beta}$. Because of the uniqueness of the extension $\widehat{\beta}$, this construction defines a functor. Moreover, $r \circ i=\operatorname{Id}_{\widehat{P} \downarrow^{\prime}}$, and $i \circ r \simeq \operatorname{Id}_{P \downarrow \iota_{N}}$, where the homotopy is induced by the natural transformation given by the inclusions $\iota_{Q}^{Q \widehat{P}}$.

As noted above, Theorem 3.5 follows immediately from Proposition 3.11 Another consequence of this proposition is the uniqueness of quasicentric linking systems associated to a given fusion system which extend a given centric linking system. In fact, in the following proposition, we prove a slightly stronger result, by comparing a more general "partial quasicentric linking system" defined on smaller sets of objects with a quasicentric linking system as constructed in Proposition 3.4 and show that the first is contained in the second if they agree after restricting to centric radical subgroups. For any $p$-local finite group $(S, \mathcal{F}, \mathcal{L}), \mathcal{L}^{r}$ denotes the full subcategory of $\mathcal{L}$ whose objects are the subgroups which are $\mathcal{F}$-radical as well as $\mathcal{F}$-centric.

Proposition 3.12. Fix a p-local finite group $(S, \mathcal{F}, \mathcal{L})$, and let $\mathcal{L}^{q}$ be the qua- 
sicentric linking system constructed in Proposition 3.4 Let $\mathcal{H}$ be any set of $\mathcal{F}$ quasicentric subgroups of $S$ which is closed under $\mathcal{F}$-conjugacy and overgroups, and which contains all $\mathcal{F}$-centric $\mathcal{F}$-radical subgroups. Let $\mathcal{L}^{\prime}$ be a category with $\operatorname{Ob}\left(\mathcal{L}^{\prime}\right)=\mathcal{H}$, together with a functor $\pi^{\prime}: \mathcal{L}^{\prime} \longrightarrow \mathcal{F}$, and distinguished monomorphisms $\delta_{P}^{\prime}$ for all $P \in \mathcal{H}$, which satisfy axioms $(A)_{q},(B)_{q},(C)_{q}$, and $(D)_{q}$ in Definition 3.3. Assume $\mathcal{L}^{\prime}$ contains a full subcategory isomorphic to $\mathcal{L}^{r}$ in a way compatible with the projection functors and distinguished monomorphisms. Then $\mathcal{L}^{\prime}$ is isomorphic to the full subcategory of $\mathcal{L}^{q}$ with object set $\mathcal{H}$, via an inclusion functor $\mathcal{L}^{\prime} \longrightarrow \mathcal{L}^{q}$ which commutes with the projection functors and distinguished monomorphisms for both categories.

Proof. By Theorem 3.5 $\left|\mathcal{L}^{\prime}\right| \simeq\left|\mathcal{L}^{r}\right| \simeq|\mathcal{L}|$. More precisely, the second equivalence follows directly from the theorem, and the first equivalence follows from the same argument applied to $\mathcal{L}^{\prime}$, since we never needed to know that the linking system was defined on all $\mathcal{F}$-quasicentric subgroups (or even on all $\mathcal{F}$-centric subgroups).

In particular, $\mathcal{L}^{q}$ is a full subcategory of

$$
\mathcal{L}_{S, f}\left(|\mathcal{L}|_{p}^{\wedge}\right) \cong \mathcal{L}_{S, f}\left(\left|\mathcal{L}^{\prime}\right|_{p}^{\wedge}\right)
$$

So if we let $\xi_{\mathcal{L}^{\prime}}: \mathcal{L}^{\prime} \longrightarrow \mathcal{L}_{S, f}\left(\left|\mathcal{L}^{\prime}\right|_{p}^{\wedge}\right)$ be the functor defined earlier in the section (just before Proposition 3.4), then $\xi_{\mathcal{L}^{\prime}}$ defines an inclusion of $\mathcal{L}^{\prime}$ into $\mathcal{L}^{q}$, which is clearly compatible with the projection functors and distinguished monomorphisms.

\section{Constrained fusion systems}

We now look at a class of saturated fusion systems which have very simple, regular behavior: the constrained fusion systems. The main results here say that constrained fusion systems are always realized as fusion systems of finite groups in a predictable way, and have unique associated centric linking systems.

Let $\mathcal{F}$ be an arbitrary saturated fusion system over a $p$-group $S$. Recall (Definition 1.5) that a subgroup $Q \triangleleft S$ is normal in $\mathcal{F}$ if each $\alpha \in \operatorname{Hom}_{\mathcal{F}}\left(P, P^{\prime}\right)$ extends to a morphism $\bar{\alpha} \in \operatorname{Hom}_{\mathcal{F}}\left(P Q, P^{\prime} Q\right)$ which sends $Q$ to itself. If $Q$ and $Q^{\prime}$ are both normal in $\mathcal{F}$, then clearly $Q Q^{\prime}$ is normal in $\mathcal{F}$. Hence, there is a unique maximal normal $p$-subgroup in $\mathcal{F}$, which we denote $O_{p}(\mathcal{F})$ by analogy with the subgroup $O_{p}(G)$ of a finite group $G$. By Proposition [1.6 $O_{p}(\mathcal{F})$ is contained in the intersection of all $\mathcal{F}$-radical subgroups of $S$. We are interested in the case when $O_{p}(\mathcal{F})$ is itself $\mathcal{F}$-centric, or equivalently, when there is a subgroup $P \triangleleft S$ which is both normal and centric in $\mathcal{F}$.

DeFinition 4.1. A saturated fusion system $\mathcal{F}$ over a p-group $S$ is constrained if there is some $Q \triangleleft S$ which is $\mathcal{F}$-centric and normal in $\mathcal{F}$.

When $G$ is a finite $p^{\prime}$-reduced group, then $G$ is said to be $p$-constrained if there exists some normal $p$-subgroup $P \triangleleft G$ which is centric in $G$ (i.e., $C_{G}(P) \leq P$ ). (More generally, an arbitrary finite group $G$ is $p$-constrained if its $p^{\prime}$-reduction $G / O_{p^{\prime}}(G)$ is $p$-constrained.) Our aim is to show that any constrained fusion system is the fusion system of a unique $p^{\prime}$-reduced $p$-constrained group $G$. This will be 
done by first showing that each constrained fusion system has a unique associated centric linking system $\mathcal{L}$, and then choosing $G$ to be a certain automorphism group in $\mathcal{L}$.

We first show that for any constrained fusion system, the obstruction groups to the existence and uniqueness of an associated centric linking system vanish. For any saturated fusion system $\mathcal{F}$, let $\mathcal{Z}_{\mathcal{F}}$ denote the functor on $\mathcal{O}\left(\mathcal{F}^{c}\right)$ defined by setting $\mathcal{Z}_{\mathcal{F}}(P)=Z(P)$ for all $\mathcal{F}$-centric $P \leq S$. (See [5] $\S 3$ ] for details.)

Proposition 4.2. Let $\mathcal{F}$ be any constrained saturated fusion system over a p-group $S$. Then

$$
\varliminf_{\mathcal{O}\left(\mathcal{F}^{c}\right)}^{i}\left(\mathcal{Z}_{\mathcal{F}}\right)=0 \quad \text { for all } i>0
$$

In particular, there is a centric linking system $\mathcal{L}$ associated to $\mathcal{F}$ which is unique up to isomorphism.

Proof. Fix $Q \triangleleft S$ which is $\mathcal{F}$-centric and normal in $\mathcal{F}$. Let $P_{1}, P_{2}, \ldots, P_{m}$ be $\mathcal{F}$ conjugacy class representatives for all $\mathcal{F}$-centric subgroups $P \leq S$ such that $P \nsupseteq Q$, arranged such that $\left|P_{i}\right| \leq\left|P_{i+1}\right|$ for each $i$. For $i=0,1, \ldots, m$, let $\mathcal{Z}_{i} \subseteq \mathcal{Z}_{\mathcal{F}}$ be the subfunctor

$$
\mathcal{Z}_{i}(P)= \begin{cases}Z(P) & \text { if } P \text { is } \mathcal{F} \text {-conjugate to } P_{j} \text { for some } j>i \\ 0 & \text { otherwise. }\end{cases}
$$

This gives a sequence of subfunctors $\mathcal{Z}_{\mathcal{F}} \supseteq \mathcal{Z}_{0} \supseteq \mathcal{Z}_{1} \supseteq \cdots \supseteq \mathcal{Z}_{m}=0$, where for each $i=1, \ldots, m, \mathcal{Z}_{i-1} / \mathcal{Z}_{i}$ vanishes except on subgroups $\mathcal{F}$-conjugate to $P_{i}$. Hence by [5. Proposition 3.2],

$$
\lim _{\mathcal{O}\left(\mathcal{F}^{c}\right)}^{*}\left(\mathcal{Z}_{i-1} / \mathcal{Z}_{i}\right) \cong \Lambda^{*}\left(\operatorname{Out}_{\mathcal{F}}\left(P_{i}\right) ; Z\left(P_{i}\right)\right)
$$

Furthermore, since $P_{i} \nsupseteq Q, N_{P_{i} Q}\left(P_{i}\right) / P_{i} \cong \operatorname{Out}_{Q}\left(P_{i}\right)$ is a nontrivial normal $p$ subgroup of $\operatorname{Out}_{\mathcal{F}}\left(P_{i}\right)$ (normal by the same argument as the one used in the proof of Proposition [1.6), $\Lambda^{*}\left(\operatorname{Out}_{\mathcal{F}}\left(P_{i}\right) ; Z\left(P_{i}\right)\right)=0$ by [14 Proposition 6.1(ii)]. This proves that $\lim ^{*}\left(\mathcal{Z}_{i}\right)=0$ for all $i$, and in particular that $\lim ^{*}\left(\mathcal{Z}_{0}\right)=0$. Thus

$$
\lim _{\mathcal{O}\left(\mathcal{F}^{c}\right)}^{*}\left(\mathcal{Z}_{\mathcal{F}}\right) \cong{\underset{\mathcal{O}\left(\mathcal{F}^{c}\right)}{\lim ^{*}}}^{*}\left(\mathcal{Z}_{\mathcal{F}} / \mathcal{Z}_{0}\right)
$$

where $\mathcal{Z}_{\mathcal{F}} / \mathcal{Z}_{0}$ is the quotient functor

$$
\left(\mathcal{Z}_{\mathcal{F}} / \mathcal{Z}_{0}\right)(P)= \begin{cases}Z(P)=Z(Q)^{P} & \text { if } P \geq Q \\ 0 & \text { if } P \nsupseteq Q .\end{cases}
$$

Now set $\Gamma=\operatorname{Out}_{\mathcal{F}}(Q)$ and $S_{0}=\operatorname{Out}_{S}(Q) \cong S / Q$. Thus $S_{0} \in \operatorname{Syl}_{p}(\Gamma)$. Set $M=Z(Q)$, regarded as a $\mathbb{Z}_{(p)}[\Gamma]$-module. Let $H^{0} M$ be the fixed-point functor on $\mathcal{O}_{S_{0}}(\Gamma)$ defined by $H^{0} M(P)=M^{P}$. Then $H^{0} M$ is acyclic by [13 Proposition 5.14] (shown more explicitly in [14 Proposition 5.2]). So by (1), we will be done upon showing that

$$
\lim _{\mathcal{O}\left(\mathcal{F}^{c}\right)} *\left(\mathcal{Z}_{\mathcal{F}} / \mathcal{Z}_{0}\right) \cong \lim _{\mathcal{O}_{S_{0}}(\Gamma)} *\left(H^{0} M\right)
$$

Since $Q$ is normal and centric in $\mathcal{F}$, it is easy to check that $\mathcal{O}_{S_{0}}(\Gamma)$ is isomorphic to 
the full subcategory of $\mathcal{O}\left(\mathcal{F}^{c}\right)$ with objects the subgroups of $S$ containing $Q$. Under this identification, $H^{0} M$ is the restriction of $\mathcal{Z}_{\mathcal{F}} / \mathcal{Z}_{0}$ by (2). Isomorphism (3) now follows since $\left(\mathcal{Z}_{\mathcal{F}} / \mathcal{Z}_{0}\right)(P)=0$ for all $P \nsupseteq Q$, and since there are no morphisms in $\mathcal{O}\left(\mathcal{F}^{c}\right)$ from an object in the subcategory to an object not in it.

The existence and uniqueness of a centric linking system associated to $\mathcal{F}$ now follow from [5, Proposition 3.1].

We are now ready to show that each constrained fusion system is the fusion system of a group. The following proposition includes Proposition $\mathrm{C}$

Proposition 4.3. Let $\mathcal{F}$ be a constrained saturated fusion system over a $p$ group $S$. Then there is a unique finite $p^{\prime}$-reduced $p$-constrained group $G$, containing $S$ as a Sylow p-subgroup, such that $\mathcal{F}=\mathcal{F}_{S}(G)$ as fusion systems over $S$. Furthermore, if $\mathcal{L}$ is a centric linking system associated to $\mathcal{F}$, then

(a) $G \cong \operatorname{Aut}_{\mathcal{L}}(Q)$ for any subgroup $Q \triangleleft S$ which is $\mathcal{F}$-centric and normal in $\mathcal{F}$; and

(b) $\mathcal{L} \cong \mathcal{L}_{S}^{c}(G)$.

Proof. Using Proposition 4.2 fix a centric linking system $\mathcal{L}$ associated to $\mathcal{F}$. Let $\pi: \mathcal{L} \longrightarrow \mathcal{F}^{c}$ denote the canonical projection functor. By Lemma 3.7 any choice of "inclusion" morphisms $\iota_{P} \in \operatorname{Mor}_{\mathcal{L}}(P, S)$ determines unique injections

$$
\delta_{P, P^{\prime}}: N_{S}\left(P, P^{\prime}\right) \longrightarrow \operatorname{Mor}_{\mathcal{L}}\left(P, P^{\prime}\right),
$$

for all $\mathcal{F}$-centric subgroups $P, P^{\prime} \leq S$, which satisfy the following conditions:

(i) $\pi\left(\delta_{P, P^{\prime}}(g)\right)=c_{g} \in \operatorname{Hom}_{\mathcal{F}}\left(P, P^{\prime}\right)$ for $g \in N_{S}\left(P, P^{\prime}\right)$;

(ii) $\delta_{P, P}(g)=\delta_{P}(g) \in \operatorname{Aut}_{\mathcal{L}}(P)$ for $g \in P$;

(iii) $\delta_{P, P^{\prime \prime}}(h g)=\delta_{P^{\prime}, P^{\prime \prime}}(h) \circ \delta_{P, P^{\prime}}(g)$ for $g \in N_{S}\left(P, P^{\prime}\right)$ and $h \in N_{S}\left(P^{\prime}, P^{\prime \prime}\right)$; and

(iv) $\delta_{P, S}(1)=\iota_{P}$.

Set $\iota_{P}^{P^{\prime}}=\delta_{P, P^{\prime}}(1) \in \operatorname{Hom}_{\mathcal{L}}\left(P, P^{\prime}\right)$ for all $P \leq P^{\prime}$ containing $Q$. We think of these as the "inclusion morphisms" in $\mathcal{L}$. By construction, $\iota_{P}^{S}=\iota_{P}$ and $\iota_{P}^{P}=\operatorname{Id}_{P}$ for all $P$, and $\iota_{P}^{P^{\prime \prime}}=\iota_{P^{\prime}}^{P^{\prime \prime}} \circ \iota_{P}^{P^{\prime}}$ whenever $P \leq P^{\prime} \leq P^{\prime \prime}$.

The proposition follows from the following points, which will be proven in Steps $1-2$.

(1) Assume $Q \triangleleft S$ is $\mathcal{F}$-centric and normal in $\mathcal{F}$, and $G=\operatorname{Aut}_{\mathcal{L}}(Q)$. Then $G$ is $p^{\prime}$-reduced and $p$-constrained; and we can identify $S$ with a subgroup of $G$ in such a way that $S \in \operatorname{Syl}_{p}(G)$ and $\mathcal{F}=\mathcal{F}_{S}(G)$.

(2) Assume $G$ is $p^{\prime}$-reduced and $p$-constrained, and such that $S \in \operatorname{Syl}_{p}(G)$ and $\mathcal{F}=\mathcal{F}_{S}(G)$. Then $\mathcal{L} \cong \mathcal{L}_{S}^{c}(G)$. Also, if $Q \triangleleft S$ is any subgroup which is $\mathcal{F}$ centric and normal in $\mathcal{F}$, then $Q \triangleleft G$, and $G \cong \operatorname{Aut}_{\mathcal{L}}(Q)$.

Step 1: Fix $Q \triangleleft S$ which is $\mathcal{F}$-centric and normal in $\mathcal{F}$, and set $G=\operatorname{Aut}_{\mathcal{L}}(Q)$. Via the injection

$$
\delta_{Q, Q}: S=N_{S}(Q) \longrightarrow \operatorname{Aut}_{\mathcal{L}}(Q)=G
$$


we identify $S$ as a subgroup of $G$. Since $Q$ is fully normalized,

$$
S / Z(Q) \cong \operatorname{Aut}_{S}(Q) \in \operatorname{Syl}_{p}\left(\operatorname{Aut}_{\mathcal{F}}(Q)\right)
$$

where $\operatorname{Aut}_{\mathcal{F}}(Q) \cong G / Z(Q)$; and thus $S \in \operatorname{Syl}_{p}(G)$.

Let $P, P^{\prime} \leq S$ be any pair of subgroups which contain $Q$. For any $f \in \operatorname{Mor}_{\mathcal{L}}\left(P, P^{\prime}\right)$, there is (by Lemma 3.6) a unique "restriction" of $f$ to $Q$ : a unique element $\gamma(f) \in$ $G=\operatorname{Aut}_{\mathcal{L}}(Q)$ such that $\iota_{Q}^{S} \circ \gamma(f)=f \circ \iota_{Q}^{P}$. These restrictions clearly satisfy the following two conditions:

(v) $\gamma\left(f^{\prime} \circ f\right)=\gamma\left(f^{\prime}\right) \cdot \gamma(f)$ for any $f^{\prime} \in \operatorname{Mor}_{\mathcal{L}}\left(P^{\prime}, P^{\prime \prime}\right)$, any $Q \leq P^{\prime \prime} \leq S$; and

(vi) $\gamma\left(\delta_{P, P^{\prime}}(x)\right)=x$ for all $x \in N_{S}\left(P, P^{\prime}\right)$.

Furthermore, by condition (C) in Definition 1.4 for each $g \in P$,

$$
\delta_{S}(\pi(f)(g)) \circ f=f \circ \delta_{P}(g) \in \operatorname{Mor}_{\mathcal{L}}(P, S) .
$$

Upon restriction to $Q$ (and applying (v) and (vi)), this gives the relation

$$
\delta_{Q, Q}(\pi(f)(g)) \circ \gamma(f)=\gamma(f) \circ \delta_{Q, Q}(g) \in \operatorname{Aut}_{\mathcal{L}}(Q)=G .
$$

In other words, under the identification $S=\delta_{Q, Q}(S) \leq \operatorname{Aut}_{\mathcal{L}}(Q)=G$, this shows that

(vii) $\gamma(f) \in N_{G}\left(P, P^{\prime}\right)$ and $c_{\gamma(f)}=\pi(f) \in \operatorname{Hom}_{\mathcal{F}}\left(P, P^{\prime}\right)$.

Now,

$$
C_{G}(Q)=\operatorname{Ker}\left[\operatorname{Aut}_{\mathcal{L}}(Q) \stackrel{\pi}{\longrightarrow} \operatorname{Aut}_{\mathcal{F}}(Q)\right]=Z(Q):
$$

the first equality by (vii) (applied with $P=P^{\prime}=Q$, so $\gamma(f)=f$ ), and the second by condition (A) in Definition 1.4 Thus $Q$ is centric in $G$. This also shows that $O_{p^{\prime}}(G)=1$ (since $\left[O_{p^{\prime}}(G), Q\right]=1$ ), and hence that $G$ is $p^{\prime}$-reduced and $p$ constrained.

We must show that $\mathcal{F}=\mathcal{F}_{S}(G)$. We first show that $\operatorname{Hom}_{\mathcal{F}}\left(P, P^{\prime}\right) \subseteq \operatorname{Hom}_{G}\left(P, P^{\prime}\right)$ for each $P, P^{\prime} \leq S$. Since $Q$ is normal in $\mathcal{F}$, each morphism in $\operatorname{Hom}_{\mathcal{F}}\left(P, P^{\prime}\right)$ extends to a morphism in $\operatorname{Hom}_{\mathcal{F}}\left(P Q, P^{\prime} Q\right)$, and hence it suffices to work with subgroups $P, P^{\prime} \geq Q$. In particular, $P$ and $P^{\prime}$ are $\mathcal{F}$-centric in this case. For any $\varphi \in \operatorname{Hom}_{\mathcal{F}}(P, S)$, and any $f \in \operatorname{Mor}_{\mathcal{L}}(P, S)$ such that $\pi(f)=\varphi, \gamma(f) \in N_{G}\left(P, P^{\prime}\right)$ and $\varphi=c_{\gamma(f)} \in \operatorname{Hom}_{G}\left(P, P^{\prime}\right)$ by (vii), and thus $\operatorname{Hom}_{\mathcal{F}}\left(P, P^{\prime}\right) \subseteq \operatorname{Hom}_{G}\left(P, P^{\prime}\right)$.

Conversely, for any $P, P^{\prime} \leq S$ and any $g \in N_{G}\left(P, P^{\prime}\right)=N_{G}\left(P Q, P^{\prime} Q\right)$, we claim that $c_{g} \in \operatorname{Hom}_{\mathcal{F}}\left(P, P^{\prime}\right)$. Again, we can assume that $P, P^{\prime} \geq Q$. Now, $\left.c_{g}\right|_{Q} \in$ Aut $_{\mathcal{F}}(Q)$ by (vii) (applied with $P=P^{\prime}=Q$ and $f=g$ ). Since $Q=g Q g^{-1}$ is $\mathcal{F}$ centric, it is fully centralized in $\mathcal{F}$, and so $\left.c_{g}\right|_{Q}$ extends to an $\mathcal{F}$-morphism defined on

$$
N_{c_{g} \mid Q} \stackrel{\text { def }}{=}\left\{x \in S \mid c_{g x g^{-1}} \in \operatorname{Aut}_{S}(Q)\right\} \geq P,
$$

by condition (II) of Definition [1.3 In particular, $\left.c_{g}\right|_{Q}$ extends to a morphism $\varphi \in$ $\operatorname{Hom}_{\mathcal{F}}(P, S) \subseteq \operatorname{Hom}_{G}(P, S)$ (where the inclusion holds by the previous paragraph). Let $h \in N_{G}(P, S)$ be such that $\varphi=c_{h}$. Then $\left.c_{h}\right|_{Q}=\left.\varphi\right|_{Q}=\left.c_{g}\right|_{Q}$, so $h=g x$ for some $x \in C_{G}(Q)$, and $C_{G}(Q)=Z(Q)$ as already shown. Since $x \in P, c_{x} \in \operatorname{Aut}_{\mathcal{F}}(P)$, so $c_{g} \in \operatorname{Hom}_{\mathcal{F}}(P, S)$, and $c_{g} \in \operatorname{Hom}_{\mathcal{F}}\left(P, P^{\prime}\right)$ since $c_{g}(P)=g P g^{-1} \leq P^{\prime}$.

Step 2: Let $G$ be any finite $p^{\prime}$-reduced $p$-constrained group such that $S \in \operatorname{Syl}_{p}(G)$ and $\mathcal{F}=\mathcal{F}_{S}(G)$. Then $\mathcal{L} \cong \mathcal{L}_{S}^{c}(G)$ by the uniqueness in Proposition 4.2

Let $Q \triangleleft S$ be any subgroup normal in $\mathcal{F}=\mathcal{F}_{S}(G)$. Set $Q^{\prime}=O_{p}(G)$; thus 
$C_{G}\left(Q^{\prime}\right)=Z\left(Q^{\prime}\right)$ by assumption. Since $Q$ is normal in $\mathcal{F}_{S}(G)$, for any $g \in G$, $c_{g} \in \operatorname{Aut}_{G}\left(Q^{\prime}\right)$ extends to some $c_{g^{\prime}} \in \operatorname{Aut}_{G}\left(Q Q^{\prime}\right)$; then $g^{-1} g^{\prime} \in C_{G}\left(Q^{\prime}\right)=Z\left(Q^{\prime}\right)$, $g^{\prime} \in N_{G}\left(Q Q^{\prime}\right)$, and so $g \in N_{G}\left(Q Q^{\prime}\right)$. This shows that $Q Q^{\prime} \triangleleft G$, a normal $p$ subgroup, and hence $Q \leq Q^{\prime}=O_{p}(G)$. Hence for any $g \in G, c_{g} \in \operatorname{Aut}_{G}\left(Q^{\prime}\right)$ restricts to an automorphism of $Q$ (since $Q$ is normal in $\mathcal{F}_{S}(G)$ ), so $g \in N_{G}(Q)$, and this shows that $Q \triangleleft G$.

In particular, if $Q$ is both $\mathcal{F}$-centric and normal in $\mathcal{F}$, then

$$
\operatorname{Aut}_{\mathcal{L}}(Q) \cong \operatorname{Aut}_{\mathcal{L}_{S}^{c}(G)}(Q) \cong N_{G}(Q) / O^{p}\left(C_{G}(Q)\right)=G / 1 \cong G .
$$

It is in general not true, for a constrained fusion system $\mathcal{F}$ over a $p$-group $S$ and a finite group $G$ such that $S \in \operatorname{Syl}_{p}(G)$ and $\mathcal{F}=\mathcal{F}_{S}(G)$, that $p$-subgroups of $S$ normal in $\mathcal{F}$ are also normal in $G$. For example, if $G=A_{5}, p=2, S \in \operatorname{Syl}_{2}(G)$, and $\mathcal{F}=\mathcal{F}_{S}(G)$, then $\mathcal{F}$ is a constrained fusion system, with $O_{2}(\mathcal{F})=S \cong C_{2}^{2}$. Thus $S$ is normal in $\mathcal{F}$, but not in $G$, in this case. This shows the importance of assuming $G$ is $p^{\prime}$-reduced and $p$-constrained. In the given example, the unique $2^{\prime}$-reduced 2-constrained group associated to $\mathcal{F}$ is $A_{4}$.

\section{References}

1. J. Alperin, Sylow intersections and fusion, J. Algebra 6 (1967), 222-241

2. J. Alperin \& M. Broué, Local methods in block theory, Annals of Math. 110 (1979), 143-157

3. C. Broto, R. Levi, On spaces of self homotopy equivalences of $p$-completed classifying spaces of finite groups and homotopy group extensions, Topology 41 (2002), 229-255

4. C. Broto, R. Levi, \& B. Oliver, Homotopy equivalences of $p$-completed classifying spaces of finite groups, Invent. math. 151 (2003), 611-664

5. C. Broto, R. Levi, \& B. Oliver, The homotopy theory of fusion systems, J. Amer. Math. Soc. 16 (2003), 779-856

6. C. Broto, N. Castellana, J. Grodal, R. Levi, \& B. Oliver, Extensions of $p$-local finite groups (in preparation)

7. C. Broto \& J. Møller, Homotopy finite Chevalley versions of $p$-compact groups (preprint)

8. W. Dwyer, Homology decompositions for classifying spaces of finite groups, Topology 36 (1997), 783-804

9. D. Goldschmidt, A conjugation family for finite groups, J. Algebra 16 (1970), 138-142

10. D. Gorenstein, Finite groups, Harper \& Row (1968)

11. J. Grodal, Higher limits via subgroup complexes, Annals of Math. 155 (2002), 405-457

12. J. Hollender \& R. Vogt, Modules of topological spaces, applications to homotopy limits and $E_{\infty}$ structures, Arch. Math. 59 (1992), 115-129

13. S. Jackowski \& J. McClure, Homotopy decomposition of classifying spaces via elementary abelian subgroups, Topology 31 (1992), 113-132

14. S. Jackowski, J. McClure, \& B. Oliver, Homotopy classification of self-maps of $B G$ via $G$ actions, Annals of Math. 135 (1992), 184-270

15. R. Levi, \& B. Oliver, Construction of 2-local finite groups of a type studied by Solomon and Benson, Geometry \& Topology 6 (2002), 917-990

16. S. Mac Lane, Homology, Springer-Verlag (1963, 1975)

17. Ll. Puig, Structure locale dans les groupes finis, Bull. Soc. Math. France Suppl. Mém. 47 (1976)

18. Ll. Puig, Unpublished notes (ca. 1990)

19. Ll. Puig, The hyperfocal subalgebra of a block, Invent. math. 141 (2000), 365-397

20. D. Quillen, Algebraic $K$-theory I, Lecture notes in mathematics 341 (1973), 77-139

21. A. Ruiz \& A. Viruel, The classification of $p$-local finite groups over the extraspecial group of order $p^{3}$ and exponent $p$, Math. Z. 248 (2004), 45-65 
C. Broto $\&$ N. Castellana

Departament de Matemàtiques Universitat Autònoma de Barcelona

E-08193 Bellaterra, Spain

broto@mat.uab.es

natalia@mat.uab.es

R. Levi

Department of Mathematical Sciences University of Aberdeen, Meston Building 339

Aberdeen AB24 3UE, U.K.

ran@maths.abdn.ac.uk
J. Grodal

Department of Mathematics University of Chicago Chicago, IL 60637, USA

jg@math.uchicago.edu

B. Oliver LAGA, Institut Galilée Av. J-B Clément 93430 Villetaneuse, France bob@math.univ-paris13.fr 